\title{
Theory of neutral and charged exciton scattering with electrons in semiconductor quantum wells
}

\author{
G. Ramon, ${ }^{1, \text { 冈 A. Mann, }}{ }^{1}$ and E. Cohen ${ }^{1,2}$ \\ ${ }^{1}$ Department of Physics, Technion - Israel Institute of Technology, Haifa 32000, Israel \\ ${ }^{2}$ Solid State Institute, Technion - Israel Institute of Technology, Haifa 32000, Israel
}

\begin{abstract}
Electron scattering on both neutral $(X)$ and charged $\left(X^{-}\right)$excitons in quantum wells is studied theoretically. A microscopic model is presented, taking into account both elastic and dissociating scattering. The model is based on calculating the exciton-electron direct and exchange interaction matrix elements, from which we derive the exciton scattering rates. We find that for an electron density of $10^{9} \mathrm{~cm}^{-2}$ in a GaAs QW at $T=5 \mathrm{~K}$, the $X^{-}$linewidth due to electron scattering is roughly twice as large as that of the neutral exciton. This reflects both the $X^{-}$larger interaction matrix elements compared with those of $X$, and their different dependence on the transferred momentum. Calculated reflection spectra can then be obtained by considering the three electronic excitations of the system, namely, the heavy-hole and light-hole 1S neutral excitons, and the heavy-hole $1 \mathrm{~S}$ charged exciton, with the appropriate oscillator strengths.
\end{abstract}

PACS numbers: 34.80.-i, 78.67.De, 73.21.Fg

\section{INTRODUCTION}

The broadening of exciton emission lines due to electran-exciton collisions was first observed in bulk GaAst. An experimental comparison between excitonelectron anf exciton-exciton scattering mechanisms in bulk GaAs a showed that the exciton-electron scattering efficiency is an order of magnitude larger than that of the excitonexciton process. Both scattering processes are enhanced for the 2D excitons as compared to bulk excitons. Theoretical calculations of elastic and inelastic exciton-electron scattering were reported for bulk semiconductors $\mathrm{E}$ and for $\mathrm{QW}$ ' $\mathrm{S}$. The semiclassical treatment of the latter neglected the exchange term which was shown recently to be the dominant term in QW'st.

The existence of the negatively charged exciton $X^{-}$ (trion) was first proposed by Lampert as the semiconductor analogue of the hydrogen ion. Due to their confinement in the growth direction, 2D trions have a binding energy which is ap order-of-magnitude larger than that of the bulk trions This fact facilitated the observation of trions in $\mathrm{CdTe}_{10}$ and in GaAs 11 QW's containing a low-density two-dimensional electron gas (2DEG). Theoretical calculations of the binding energy of both negatively $\left(X^{-}\right)$and positively $\left(X^{+}\right)$charged excitons were performed using various trial functions, showing that only the singlet state is bound 12 13. Particular attention was devoted to the modification of the trion's properties in the presence of strong magnetic fields. In this limit, the $X^{-}$binding energy is increased, and the electron spin triplet state becomes bound as well14. It was also observed that the dependence on the magnetic field of the $X^{-}$and $X^{+}$binding energies, as well as their Zeeman splitting, differ drastically (while being nearly identical at zero magnetic field) 15 .

While these aspects of the trions where extensively studied, there are very few reports on their broadening mechanisms 16] In this paper we present a theoretical model for the scattering of both neutral and charged excitons with electrons in QW's. This paper continues our previous work which introduced neutral excitons elastic scattering with electrons in the context of cavity polaritons 7 . Here we extend our model, to incorporate both excitons and trions, taking into account elastic and inelastic scattering processes. The model is based on calculating the exciton-electron direct and exchange interaction matrix elements, from which we derive the exciton scattering rates. These are integrated over all final excitonic states, resulting in the exciton linewidth due to scattering, as a function of its initial momentum (or energy). A major difference between the exciton and trion scattering lies in the charge of the latter, which results in a divergence of its matrix elements in the limit of zero transferred momentum. This divergence, originating from the infinite range of the Coulomb potential, is treated by applying the Lindhard model for the potential screening. The screening action complicates the trion linewidth dependence on the electron density; thus, while for electron densities larger than $5 \cdot 10^{9} \mathrm{~cm}^{-2}$ the trion linewidth due to electron scattering in a GaAs QW at $T=5 \mathrm{~K}$ is comparable to that of the neutral exciton, for a very dilute $2 \mathrm{DEG}\left(n_{\mathrm{e}} \approx 5 \cdot 10^{8} \mathrm{~cm}^{-2}\right)$ the trion linewidth becomes roughly an order-of-magnitude larger than that of the neutral exciton. Inelastic scattering is also much more efficient in the trion's case, due to its smaller binding energy. The calculated reflection spectra are obtained by considering the three electronic excitations of the system, namely, the heavy-hole and light-hole 1S neutral excitons, and the heavy-hole 1S charged exciton (trion), with the appropriate oscillator strengths. A qualitative validation of our calculations is given by considering photoluminesence (PL) measurements that were done on a mixed type I - type II GaAs/AlAs QW (MTQW) structure17.

The paper is organized as follows. In section II, we present in detail the model for the neutral exciton - elec- 
tron scattering. In section III we consider the trionelectron scattering. First we construct the trion's wave function, which is used in the matrix elements calculations. We then treat the divergences that originate from the infinite range of the Coulomb potential, by applying the Lindhard model for the potential screening. The effect of the screening is discussed in the context of the trion linewidth dependence on the electron density. In section [IV] we use our model to calculate QW reflection spectra and discuss their relevance to the available experimental data. A summary and conclusions are given in section $\mathrm{V}$. In Appendices A and B, we provide some details of the direct and exchange integrals calculations for the neutral exciton - electron scattering. Finally, a derivation of the trion binding energy, using a Chandrasekhar-type trial function, is given in Appendix C.

\section{A MICROSCOPIC MODEL FOR EXCITON - ELECTRON SCATTERING}

In this section we present a detailed description of the exciton-electron scattering, considering separately the elastic process, for which the exciton remains bound, and the inelastic process, where the exciton breaks into an unbound electron-hole pair.

\section{A. Elastic scattering}

We first construct the state of a single exciton with an in-plane center-of-mass (CM) momentum $\mathbf{k}_{\mathrm{x}}$ in the fermionic Hilbert space of electron-hole pairs, using the notations of Tassone and Yamamoto 18 . It is a superposition of wave functions with different electron momenta $\mathbf{k}_{1}$ and electron and hole $z$ coordinates, given by

$$
\left|\mathbf{k}_{\mathbf{x}}\right\rangle=\sum_{\mathbf{k}_{1}} \int d z_{e} d z_{h} \phi_{\alpha \mathbf{k}_{\mathbf{x}}+\mathbf{k}_{1}}^{*}\left(z_{e}, z_{h}\right) c_{-\mathbf{k}_{1}, z_{e}}^{\dagger} d_{\mathbf{k}_{\mathbf{x}}+\mathbf{k}_{1}, z_{h}}^{\dagger}|0\rangle,
$$

where $c_{\mathbf{k}_{\mathbf{x}}, z_{e}}^{\dagger}\left(d_{\mathbf{k}_{\mathbf{x}}, z_{h}}^{\dagger}\right)$ is the electron (hole) creation operator with in-plane momentum $\mathbf{k}_{\mathrm{x}}$ and $z_{e}\left(z_{h}\right)$ coordinate, $m_{\mathrm{e}}, M_{\mathrm{x}}$ are the electron and exciton in-plane effective masses, respectively, $\alpha=m_{\mathrm{e}} / M_{\mathrm{x}}$, and $\phi_{\mathbf{k}}\left(z_{e}, z_{h}\right)=$ $\mathcal{N}\left[1+(\lambda k)^{2}\right]^{-3 / 2} \chi_{e}\left(z_{e}\right) \chi_{h}\left(z_{h}\right)$ is the in-plane Fourier transform of the exciton wave function (see Appendix A). A state comprising an exciton and an unbound electron having $\mathbf{k}_{\mathrm{e}}$ and $z_{c}$ will be written as

$$
\begin{aligned}
\left|\mathbf{k}_{\mathbf{x}} ; \mathbf{k}_{\mathrm{e}}\right\rangle= & \sum_{\mathbf{k}_{1}} \int d z_{e} d z_{h} d z_{c} \phi_{\alpha \mathbf{k}_{\mathrm{x}}+\mathbf{k}_{1}}^{*}\left(z_{e}, z_{h}\right) \psi_{\mathbf{k}_{\mathrm{e}}}^{*}\left(z_{c}\right) \times \\
& c_{-\mathbf{k}_{1}, z_{e}}^{\dagger} d_{\mathbf{k}_{\mathrm{x}}+\mathbf{k}_{1}, z_{h}}^{\dagger} c_{\mathbf{k}_{\mathrm{e}}, z_{c}}^{\dagger}|0\rangle
\end{aligned}
$$

where $\psi_{\mathbf{k}_{\mathrm{e}}}\left(z_{c}\right)$ is the electron wave function. Applying the Coulomb interaction operators $V_{e e}, V_{e h}$ to this state, we have for the electron-hole and electron-electron interactions:

$$
\begin{aligned}
V_{e h}\left|\mathbf{k}_{\mathbf{x}} ; \mathbf{k}_{\mathrm{e}}\right\rangle & =\sum_{\mathbf{k}_{1}, \mathbf{q}_{1}} \int d z_{e} d z_{h} d z_{c} V_{\mathbf{q}_{1}}\left(z_{h}-z_{c}\right)\left\{\phi_{\alpha \mathbf{k}_{\mathbf{x}}+\mathbf{k}_{1}+\mathbf{q}_{1}}^{*}\left(z_{e}, z_{h}\right) \psi_{\mathbf{k}_{\mathrm{e}}}^{*}\left(z_{c}\right) c_{-\mathbf{k}_{1}, z_{e}}^{\dagger} d_{\mathbf{k}_{\mathbf{x}}+\mathbf{k}_{1}, z_{h}}^{\dagger} c_{\mathbf{k}_{\mathrm{e}}, z_{c}}^{\dagger}\right. \\
& \left.+\phi_{\alpha \mathbf{k}_{\mathbf{x}}+\mathbf{k}_{1}}^{*}\left(z_{e}, z_{h}\right) \psi_{\mathbf{k}_{\mathrm{e}}}^{*}\left(z_{c}\right) c_{-\mathbf{k}_{1}, z_{e}}^{\dagger} d_{\mathbf{k}_{\mathbf{x}}+\mathbf{k}_{1}-\mathbf{q}_{1}, z_{h}}^{\dagger} c_{\mathbf{k}_{\mathrm{e}}+\mathbf{q}_{1}, z_{c}}^{\dagger}\right\}|0\rangle . \\
V_{e e}\left|\mathbf{k}_{\mathbf{x}} ; \mathbf{k}_{\mathrm{e}}\right\rangle & =\sum_{\mathbf{k}_{1}, \mathbf{q}_{1}} \int d z_{e} d z_{h} d z_{c} V_{\mathbf{q}_{1}}\left(z_{e}-z_{c}\right) \phi_{\alpha \mathbf{k}_{\mathbf{x}}+\mathbf{k}_{1}}^{*}\left(z_{e}, z_{h}\right) \psi_{\mathbf{k}_{\mathrm{e}}}^{*}\left(z_{c}\right) c_{-\mathbf{k}_{1}-\mathbf{q}_{1}, z_{e}}^{\dagger} d_{\mathbf{k}_{\mathbf{x}}+\mathbf{k}_{1}, z_{h}}^{\dagger} c_{\mathbf{k}_{\mathrm{e}}+\mathbf{q}_{1}, z_{c}}^{\dagger}|0\rangle .
\end{aligned}
$$

The first term in Eq. (II.3a) represents the Coulomb interaction between the constituents of the exciton, thus contributing to its self energy, and can be discarded in the calculation of the scattering matrix elements. In Eqs. (II.3a) and (II.3b), $V_{\mathbf{q}_{1}}(z)= \pm \frac{2 \pi e^{2}}{A \epsilon_{0} q_{1}} \mathrm{e}^{-q_{1} z}$ is the 2-dimensional Fourier transform of the Coulomb interaction, where $A$ is the QW area, and the plus (minus) sign is used in $V_{e e}\left(V_{e h}\right)$. Using the anticommutation relations for the fermion operators, one can easily find the scattering matrix elements to be

$$
\begin{aligned}
\left\langle\mathbf{k}_{\mathrm{x}}+\mathbf{q} ; \mathbf{k}_{\mathrm{e}}-\mathbf{q}\left|V_{e h}\right| \mathbf{k}_{\mathrm{x}} ; \mathbf{k}_{\mathrm{e}}\right\rangle= & \sum_{\mathbf{k}_{1}} \int d z_{e} d z_{h} d z_{c}\left[V_{\mathbf{q}}\left(z_{h}-z_{c}\right) \phi_{\alpha\left(\mathbf{k}_{\mathrm{x}}+\mathbf{q}\right)+\mathbf{k}_{1}}\left(z_{e}, z_{h}\right) \phi_{\alpha \mathbf{k}_{\mathrm{x}}+\mathbf{k}_{1}}^{*}\left(z_{e}, z_{h}\right) \psi_{\mathbf{k}_{\mathrm{e}}-\mathbf{q}}\left(z_{c}\right) \psi_{\mathbf{k}_{\mathrm{e}}}^{*}\left(z_{c}\right)-\right. \\
& \left.V_{\mathbf{k}_{1}+\mathbf{k}_{\mathrm{e}}-\mathbf{q}}\left(z_{h}-z_{c}\right) \phi_{\alpha \mathbf{k}_{\mathrm{x}}+\mathbf{k}_{1}-\beta \mathbf{q}}\left(z_{c}, z_{h}\right) \phi_{\alpha \mathbf{k}_{\mathrm{x}}+\mathbf{q}-\mathbf{k}_{\mathrm{e}}}^{*}\left(z_{e}, z_{h}\right) \psi_{\mathbf{k}_{\mathrm{e}}-\mathbf{q}}\left(z_{e}\right) \psi_{\mathbf{k}_{\mathrm{e}}}^{*}\left(z_{c}\right)\right]
\end{aligned}
$$




$$
\begin{aligned}
\left\langle\mathbf{k}_{\mathrm{x}}+\mathbf{q} ; \mathbf{k}_{\mathrm{e}}-\mathbf{q}\left|V_{e e}\right| \mathbf{k}_{\mathrm{x}} ; \mathbf{k}_{\mathrm{e}}\right\rangle= & \sum_{\mathbf{k}_{1}} \int d z_{e} d z_{h} d z_{c}\left[V_{\mathbf{q}}\left(z_{e}-z_{c}\right) \phi_{\alpha \mathbf{k}_{\mathrm{x}}-\beta \mathbf{q}+\mathbf{k}_{1}}\left(z_{e}, z_{h}\right) \phi_{\alpha \mathbf{k}_{\mathrm{x}}+\mathbf{k}_{1}}^{*}\left(z_{e}, z_{h}\right) \psi_{\mathbf{k}_{\mathrm{e}}-\mathbf{q}}\left(z_{c}\right) \psi_{\mathbf{k}_{\mathrm{e}}}^{*}\left(z_{c}\right)-\right. \\
& \left.V_{\mathbf{k}_{1}+\mathbf{k}_{\mathrm{e}}-\mathbf{q}}\left(z_{e}-z_{c}\right) \phi_{\alpha \mathbf{k}_{\mathrm{x}}-\beta \mathbf{q}+\mathbf{k}_{1}}\left(z_{c}, z_{h}\right) \phi_{\alpha \mathbf{k}_{\mathrm{x}}+\mathbf{k}_{1}}^{*}\left(z_{e}, z_{h}\right) \psi_{\mathbf{k}_{\mathrm{e}}-\mathbf{q}}\left(z_{e}\right) \psi_{\mathbf{k}_{\mathrm{e}}}^{*}\left(z_{c}\right)\right],
\end{aligned}
$$

where we denote $\beta=1-\alpha$. The first term in each of Eqs. (II.4) contributes to the direct (classical) Coulomb interaction and the second contributes to the exchange matrix element. The direct term is calculated analytically (the details can be found in Appendix A), resulting in:

$$
V_{\operatorname{dir}}(q)=\frac{4 \pi e^{2} \lambda^{3}}{\epsilon_{0} A L^{2}} h(\lambda q)[g(\lambda \beta q / 2)-g(\lambda \alpha q / 2)],
$$

where $L$ is the QW width, $\lambda$ is a variational parameter associated with the exciton Bohr radius in the QW, and we have defined the dimensionless functions

$$
\begin{aligned}
& g(\lambda q)=\left[1+(\lambda q)^{2}\right]^{-3 / 2} \\
& h(\lambda q)=\frac{\mathrm{e}^{-L q}-1+L q+\frac{5(L q)^{3}}{8 \pi^{2}}+\frac{3(L q)^{5}}{32 \pi^{4}}}{(\lambda q)^{3}\left[1+(L q / 2 \pi)^{2}\right]^{2}} .
\end{aligned}
$$

It is evident from Eq. (II.5) that the direct term is identically zero for equal electron and hole masses $(\alpha=\beta=$ $0.5)$. Similarly, we have for the exchange term

$$
\begin{gathered}
V_{\mathrm{exc}}(\Delta \mathbf{k}, \mathbf{q})=-\frac{8 e^{2} \lambda^{5}}{\epsilon_{0} A L^{2}} \int d^{2} k_{1} g\left(\lambda\left|\mathbf{k}_{1}+\alpha \mathbf{q}-\Delta \mathbf{k}\right|\right) \times \\
{\left[g\left(\lambda\left|\mathbf{k}_{1}+\mathbf{q}-\Delta \mathbf{k}\right|\right)-g(\lambda|\mathbf{q}-\Delta \mathbf{k}|)\right] h\left(\lambda k_{1}\right), \quad \text { (II.7) }}
\end{gathered}
$$

where we have defined $\Delta \mathbf{k}=\mathbf{k}_{\mathrm{e}}-\alpha \mathbf{k}_{\mathrm{x}}$. The exchange term (II.7) is computed numerically, however its angular part can be calculated analytically (see Appendix B). It is convenient to transform to dimensionless direct and exchange integrals given by

$$
V=\frac{2}{\pi} \frac{e^{2} \lambda^{3}}{\epsilon_{0} A L^{2}} I
$$

The direct and exchange integrals are plotted in Fig. 1 as a function of the transferred momentum $q$, for the case $\Delta \mathbf{k}=0$, where the angular dependence of $I_{\mathrm{exc}}$ disappears. As $q \rightarrow 0$ the direct integral approaches zero , while the exchange integral has its maximmm (this is also the case for exciton-exciton interaction 19 ). In the general case, the exchange integral is a function of the transferred momentum $q$, the momentum difference $\Delta k$ (which can be regarded, for convenience, as the in-plane momentum of the colliding electron in the rest frame of the exciton), and of the angle $\theta=\angle(\mathbf{q}, \Delta \mathbf{k})$. The exchange interaction term has the following features: (i) The interaction favors the case $\mathbf{q}=\Delta \mathbf{k}$. Physically, this means that the electron is inclined to transfer as much momentum as possible to the exciton, preferably in the same direction. (ii) The interaction retains its strength for quite large values of $q$ (or $\Delta k$ ) even though the excitonic wave function vanishes much more rapidly with momentum. (iii) The differential cross section is largest for $\theta=0$ and decreases to a minimum for back scattering $(\theta=\pi)$.

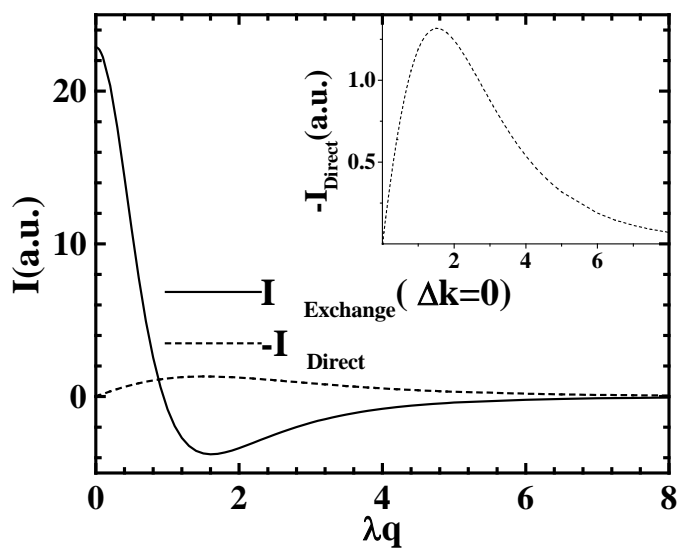

FIG. 1: Calculated direct and exchange integrals vs. transferred momentum. The inset shows $-I_{\text {dir }}$ on an expanded scale.

Next we calculate the scattering rate of an exciton, with an initial in-plane momentum $\mathbf{k}_{\mathbf{x}}$, to an excitonic state with an in-plane momentum $\mathbf{k}_{\mathrm{x}}+\mathbf{q}$. The exchange matrix element (II.7) depends on five variables: $k_{\mathrm{x}}, k_{\mathrm{e}}, q, \cos \theta, \cos \phi$, where we have defined $\phi=$ $\angle\left(\mathbf{k}_{\mathrm{x}}, \mathbf{k}_{\mathrm{x}}+\mathbf{q}\right)$. It is convenient to transform from the angles $\theta$ and $\phi$ to the angles $\gamma=\angle\left(\mathbf{k}_{\mathrm{x}}, \mathbf{q}\right)$ and $\delta=$ $\angle\left(\mathbf{k}_{\mathrm{e}}, \mathbf{q}\right) 20$ :

$$
\begin{aligned}
\cos \theta & =\frac{k_{\mathrm{e}} \cos \delta-\alpha k_{\mathrm{x}} \cos \gamma}{\sqrt{k_{\mathrm{e}}^{2}+\alpha^{2} k_{\mathrm{x}}^{2}-2 \alpha k_{\mathrm{x}} k_{\mathrm{e}} \cos (\gamma \pm \delta)}}, \quad(\Delta k \neq 0) \\
\cos \phi & =\frac{k_{\mathrm{x}}+q \cos \gamma}{\sqrt{k_{\mathrm{x}}^{2}+q^{2}+2 k_{\mathrm{x}} q \cos \gamma}}, \quad\left(\mathbf{k}_{\mathrm{x}} \neq-\mathbf{q}\right) .
\end{aligned}
$$

It can be verified that for the special case of $k_{\mathrm{x}}=0$, we have $\theta=\delta$ and $\phi=\gamma$. Conservation of energy and momentum for the exciton-electron scattering process reads

$$
E_{\mathrm{x}}\left(\left|\mathbf{k}_{\mathrm{x}}+\mathbf{q}\right|\right)+E_{\mathrm{e}}\left(\left|\mathbf{k}_{\mathrm{e}}-\mathbf{q}\right|\right)=E_{\mathrm{x}}\left(k_{\mathrm{x}}\right)+E_{\mathrm{e}}\left(k_{\mathrm{e}}\right),
$$

where $E_{\mathrm{x}}\left(E_{\mathrm{e}}\right)$ is the exciton (electron) kinetic energy. This equation is satisfied with:

$$
k_{\mathrm{e}}^{*}=\frac{1}{2 M_{\mathrm{x}} \cos \delta}\left[q\left(M_{\mathrm{x}}+m_{\mathrm{e}}\right)+2 k_{\mathrm{x}} m_{\mathrm{e}} \cos \gamma\right],
$$

The scattering rate is then found using Fermi's golden rule 
The scattering rate is then found using Fermi's golden rule

$$
\begin{aligned}
w_{\mathrm{x}-\mathrm{e}}\left(\mathbf{k}_{\mathrm{x}} \rightarrow \mathbf{k}_{\mathrm{x}}+\mathbf{q}\right) & =\frac{A}{\pi \hbar} \int d^{2} k_{\mathrm{e}} \frac{\left|V_{\mathrm{dir}}+V_{\mathrm{exc}}\right|^{2} \delta\left(k_{\mathrm{e}}-k_{\mathrm{e}}^{*}\right)}{\left|\frac{d E_{\mathrm{e}}\left(\left|\mathbf{k}_{\mathrm{e}}-\mathbf{q}\right|\right)}{d k_{\mathrm{e}}}-\frac{d E_{\mathrm{e}}\left(k_{\mathrm{e}}\right)}{d k_{\mathrm{e}}}\right|} f_{k_{\mathrm{e}}=k_{\mathrm{e}}^{*}} f_{\mathrm{fd}}\left(k_{\mathrm{e}}\right)\left[1-f_{\mathrm{fd}}\left(\left|\mathbf{k}_{\mathrm{e}}-\mathbf{q}\right|\right)\right] \\
& =\frac{A m_{e}}{\pi \hbar^{3}} \int_{0}^{2 \pi} \frac{d \delta}{|\cos \delta|} \frac{k_{\mathrm{e}}^{*}}{q}\left|V_{\mathrm{dir}}+V_{\mathrm{exc}}\right|^{2} f_{\mathrm{fd}}\left(k_{\mathrm{e}}^{*}\right)\left[1-f_{\mathrm{fd}}\left(\left|\mathbf{k}_{\mathrm{e}}^{*}-\mathbf{q}\right|\right)\right],
\end{aligned}
$$

where $f_{\mathrm{fd}}\left(k_{\mathrm{e}}\right)=\left[\mathrm{e}^{\left(E_{\mathrm{e}}\left(k_{\mathrm{e}}\right)-\mu\right) / k_{B} T}+1\right]^{-1}$ is the electron Fermi-Dirac distribution function. In the two dimensional case that we are considering, the chemical potential is $\mu=k_{B} T \log \left(\mathrm{e}^{E_{f} / k_{B} T}-1\right)$, and we assume that the Fermi energy is given by the free $2 \mathrm{D}$ electrons value: $E_{f}=\pi \hbar^{2} n_{\mathrm{e}} / m_{e}$. We note that in the high temperature limit, $E_{f} \ll k_{B} T$, the Fermi-Dirac distribution is practically classical, and that for low electron densities, the chemical potential becomes negative.

Using the relations (III.8), the integrand in (II.11) can be expressed as a function of the variables: $k_{\mathrm{x}}, q, \cos \gamma, \cos \delta$. The exciton linewidth due to electron scattering is calculated by integrating over all final excitonic states

$$
\Gamma_{\mathrm{x}-\mathrm{e}}\left(k_{\mathrm{x}}\right)=\frac{\hbar A}{(2 \pi)^{2}} \int q d q d \gamma w_{\mathrm{x}-\mathrm{e}}\left(k_{\mathrm{x}}, q, \cos \gamma\right) .
$$

Figures 2 a,b show the heavy-hole exciton $\left(M_{\mathrm{x}}=\right.$ $\left.0.177 m_{e}\right)$ and light-hole exciton $\left(M_{\mathrm{x}}=0.306 m_{e}\right)$ linewidths at $T=80 K$ and $T=5 K$ for $n_{\mathrm{e}}=5 \cdot 10^{9} \mathrm{~cm}^{-2}$. The large linewidths obtained for relatively low $n_{\mathrm{e}}$ reflect the high efficiency of the electron scattering mechanism. This should be compared to an exciton linewidth of $\approx$ $0.2 \mathrm{meV}$ for acoustic phonon scattering at $T=80 \mathrm{~K}$. The larger linewidth obtained at $T=5 K$ (Fig. 2 $2 \mathrm{~b}$ ) is explained by noticing that the exciton-electron interaction matrix elements favor small energy transfer transitions. Thus, at high temperatures, the electrons have too high energy to be effective scatterers. Decreasing $T$ thus increases the scattering rate until the electron gas becomes degenerate, and fewer final states are available for the scattered electrons. In figure 20 the heavy hole exciton linewidth at $k_{\mathrm{x}}=0$ is plotted as a function of $n_{\mathrm{e}}$ for $T=5 K$ and $80 K$ (the light hole exciton linewidth exhibits a similar behaviour). It is seen that while at $T=80 K$ the linewidth amplitudes scale practically linearly with $n_{e}$ for a large range of electron densities (up to $n_{\mathrm{e}} \approx 10^{11} \mathrm{~cm}^{-2}$ ), the linearity region at $T=5 K$ is much smaller. This linearity threshold seems to be in accord with the onset of the phase-space filling effect which becomes noticeable at higher densities and effectively enlarges the exciton Bohr radius21. As the temperature decreases, the effect of phase-space filling becomes important at a much lower electron density. We note that the functional dependence of the linewidths on the initial exciton momentum is almost unchanged within the linear regime. Increasing $n_{\mathrm{e}}$ further results in a shift of the maximum linewidth from $k_{\mathrm{x}}=0$ to higher momenta.
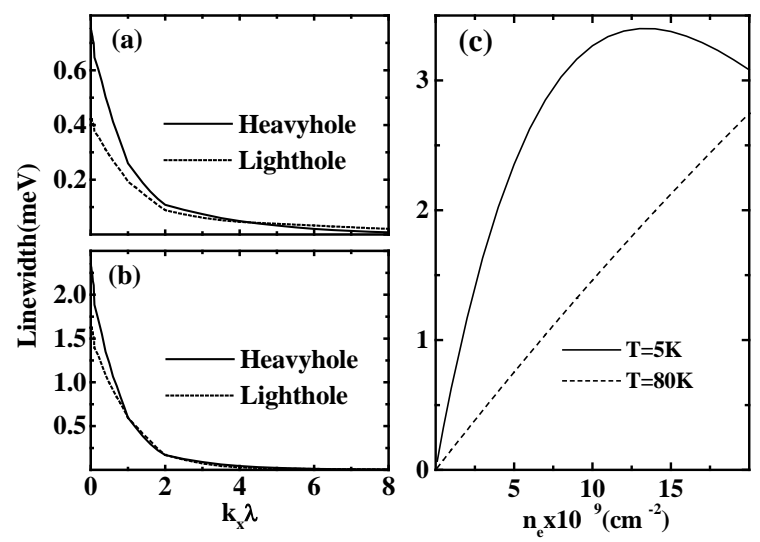

FIG. 2: (a) Heavy exciton $\left(M_{\mathrm{x}}=0.177 m_{e}\right)$ and light exciton $\left(M_{\mathrm{x}}=0.306 m_{e}\right)$ linewidths due to electron scattering for a 2DEG with $n_{\mathrm{e}}=5 \cdot 10^{9} \mathrm{~cm}^{-2}$, as a function of the exciton initial in-plane momentum, at $T=80 K$; (b) Same as (a) at $T=5 K ;(\mathrm{c})$ Linewidth of heavy hole exciton with initial momentum $k_{\mathrm{x}}=0$, as a function of $n_{\mathrm{e}}$, for the two temperatures considered.

\section{B. Exciton Dissociation scattering}

We now consider the case when the electron-exciton scattering results in dissociation. This scattering process is depicted schematically in Fig. 3. The initial state of

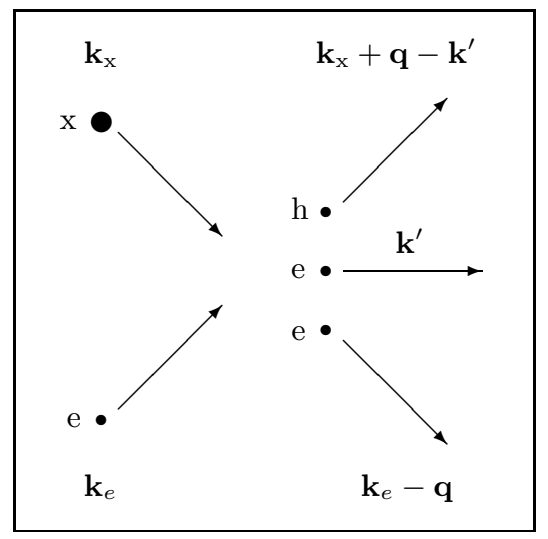

FIG. 3: Electron-exciton inelastic scattering scheme

the system is given again by Eq. (II.2) while the final 
state is that of three free particles, symbolically written as $\left|\mathbf{k}_{\mathrm{e}}-\mathbf{q} ; \mathbf{k}^{\prime} ; \mathbf{k}_{\mathrm{x}}+\mathbf{q}-\mathbf{k}^{\prime}\right\rangle$, where $\mathbf{q}$ is the momentum transferred from the free electron to the electron-hole pair, and $\mathbf{k}^{\prime}$ is the second electron momentum. Calculating the Coulomb interaction matrix elements between the initial and final states of the system, we again obtain direct and exchange interaction terms:

$$
\begin{aligned}
& V_{\text {dir }}^{\text {dis }}\left(\mathbf{k}_{\mathrm{x}}, \mathbf{q}, \mathbf{k}^{\prime}\right)=\frac{4 e^{2} \lambda^{4}}{\epsilon_{0} L^{2}}\left(\frac{2 \pi}{A}\right)^{3 / 2} h(\lambda q) \times \\
& {\left[g\left(\lambda\left|\alpha \mathbf{k}_{\mathrm{x}}+\mathbf{q}-\mathbf{k}^{\prime}\right|\right)-g\left(\lambda\left|\alpha \mathbf{k}_{\mathrm{x}}-\mathbf{k}^{\prime}\right|\right)\right] \quad(\mathrm{II} .13 \mathrm{a})} \\
& V_{\mathrm{exc}}^{\mathrm{dis}}\left(\mathbf{k}_{\mathrm{x}}, \mathbf{q}, \mathbf{k}^{\prime}, \mathbf{k}_{\mathrm{e}}\right)=-\frac{4 e^{2} \lambda^{4}}{\epsilon_{0} L^{2}}\left(\frac{2 \pi}{A}\right)^{3 / 2} h\left(\lambda\left|\mathbf{k}^{\prime}-\mathbf{k}_{\mathrm{e}}\right|\right) \times \\
& {\left[g\left(\lambda\left|\alpha \mathbf{k}_{\mathrm{x}}+\mathbf{q}-\mathbf{k}^{\prime}\right|\right)-g\left(\lambda\left|\alpha \mathbf{k}_{\mathrm{x}}+\mathbf{q}-\mathbf{k}_{\mathrm{e}}\right|\right)\right](\mathrm{II} .13 \mathrm{~b})}
\end{aligned}
$$

where $g(\lambda q), h(\lambda q)$ are given in (II.6). We note that contrary to the elastic scattering case, here the direct and exchange matrix elements are comparable. In order to calculate the scattering rate we first introduce energymomentum conservation

$$
\begin{aligned}
E_{\mathrm{x}}\left(k_{\mathrm{x}}\right)+E_{\mathrm{e}}\left(k_{\mathrm{e}}\right)= & E_{\mathrm{b}}+E_{\mathrm{h}}\left(\left|\mathbf{k}_{\mathrm{x}}+\mathbf{q}-\mathbf{k}^{\prime}\right|\right)+ \\
& E_{\mathrm{e}}\left(k^{\prime}\right)+E_{\mathrm{e}}\left(\left|\mathbf{k}_{\mathrm{e}}-\mathbf{q}\right|\right),
\end{aligned}
$$

where $E_{\mathrm{b}}$ is the exciton binding energy. Denoting the angle $\nu=\angle\left(\mathbf{k}_{\mathrm{x}}, \mathbf{k}^{\prime}\right)$ together with $\gamma, \delta$ which were defined previously, this equation is satisfied with

$$
\begin{array}{r}
k_{\mathrm{e}}^{*}=\frac{1}{2 \beta q \cos \delta}\left\{\frac{2 \beta E_{\mathrm{b}} m_{\mathrm{e}}}{\hbar^{2}}+\left(\alpha k_{\mathrm{x}}\right)^{2}+q^{2}+k^{\prime 2}+\right. \\
\left.2 \alpha\left[k_{\mathrm{x}} q \cos \gamma-k^{\prime} k_{\mathrm{x}} \cos \nu-k^{\prime} q \cos (\gamma-\nu)\right]\right\}(\mathrm{II}
\end{array}
$$

The scattering rate is then calculated to be:

$$
\begin{aligned}
& w_{\mathrm{x}-\mathrm{e}}^{\mathrm{dis}}\left(\mathbf{k}_{\mathrm{x}} \rightarrow \mathbf{k}_{\mathrm{x}}+\mathbf{q}\right)=\frac{A^{2} m_{\mathrm{e}}}{4 \pi^{3} \hbar^{3}} \int \frac{k^{\prime} d k^{\prime} d \nu d \delta}{|\cos \delta|} \frac{k_{\mathrm{e}}^{*}}{q} \times \\
& \left|V_{\mathrm{dir}}^{\mathrm{dis}} \pm V_{\mathrm{exc}}^{\mathrm{dis}}\right|^{2} f_{\mathrm{fd}}\left(k_{\mathrm{e}}^{*}\right)\left[1-f_{\mathrm{fd}}\left(\left|\mathbf{k}_{\mathrm{e}}^{*}-\mathbf{q}\right|\right)\right] \times(\mathrm{II} \\
& {\left[1-f_{\mathrm{fd}}\left(k^{\prime}\right)\right],}
\end{aligned}
$$

where the $+(-)$ sign between the direct and exchange matrix elements corresponds to the singlet (triplet) electron spin configuration. For an unpolarized electron gas, an averaging over the two spin configurations must be performed, in order to take into account the contributions of all the electrons to the exciton linewidth due to dissociating scattering:

$$
\Gamma_{\mathrm{x}-\mathrm{e}}^{\mathrm{dis}}\left(k_{\mathrm{x}}\right)=\frac{3}{4} \Gamma_{\mathrm{x}-\mathrm{e}}^{\mathrm{dis},-}\left(k_{\mathrm{x}}\right)+\frac{1}{4} \Gamma_{\mathrm{x}-\mathrm{e}}^{\mathrm{dis},+}\left(k_{\mathrm{x}}\right)
$$

$\Gamma_{\mathrm{x}-\mathrm{e}}^{\mathrm{dis},+}\left(k_{\mathrm{x}}\right)\left(\Gamma_{\mathrm{x}-\mathrm{e}}^{\mathrm{dis},-}\left(k_{\mathrm{x}}\right)\right)$ denotes the exciton linewidth contribution from the singlet (triplet) spin configuration, and both are calculated using Eq. (II.12). We note that the spin configuration averaging is irrelevant in the elastic scattering case, since the direct term there is much smaller than the exchange term. This results in similar contributions from both singlet and triplet configurations.

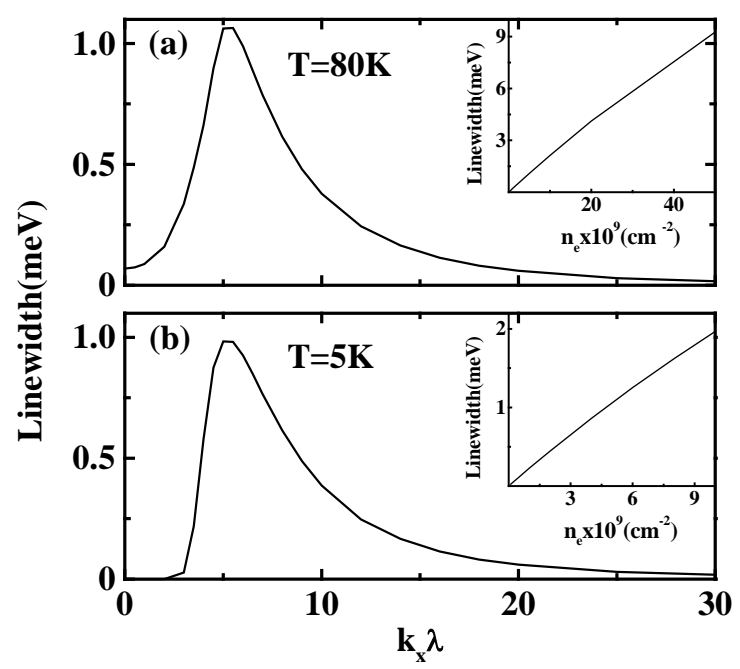

FIG. 4: Heavy exciton $\left(M_{\mathrm{x}}=0.177 m_{e}\right)$ linewidths due to dissociating scattering with electrons, as a function of exciton initial in-plane momentum, with $n_{\mathrm{e}}=5 \cdot 10^{9} \mathrm{~cm}^{-2}$, at (a) $T=80 K$; (b) $T=5 K$. The insets show the maximum linewidth dependence on $n_{\mathrm{e}}$.

Figure 1 a shows the heavy hole exciton linewidth due to dissociating scattering at $n_{\mathrm{e}}=5 \cdot 10^{9} \mathrm{~cm}^{-2}$, and $T=80 K$. Although the magnitude of $\Gamma_{\mathrm{x}-\mathrm{e}}^{\mathrm{dis}}\left(k_{\mathrm{x}}\right)$ is of the same order as the elastic scattering linewidth (compare to Fig. 2a), its functional dependence on the exciton inplane momentum is very different. In particular, we note that the maximal linewidth is obtained at a very large momentum $\left(k_{\mathrm{x}} \approx 5 \lambda^{-1}\right)$. This is due to the fact that in order for an exciton with initially small $k_{\mathrm{x}}$ to be ionized, it must scatter on an electron with energy large enough to overcome its binding energy. This is less likely as the temperature decreases, as is evident from Fig. Thb, where $\Gamma_{\mathrm{x}-\mathrm{e}}^{\mathrm{dis}}\left(k_{\mathrm{x}}\right)$ is plotted at $T=5 \mathrm{~K}$. The insets in Figure 1 show the maximum values of $\Gamma_{\mathrm{x}-\mathrm{e}}^{\mathrm{dis}}\left(k_{\mathrm{x}}\right)$ vs. $n_{\mathrm{e}}$. As in the elastic scattering case, the linear dependence on $n_{\mathrm{e}}$ holds for much larger electron densities at $T=80 \mathrm{~K}$.

\section{CHARGED EXCITON (TRION)-ELECTRON SCATTERING}

At low temperatures and low $n_{\mathrm{e}}$, charged excitons are formed. In this section we calculate the trion linewidth due to scattering with free electrons. Three scattering processes are considered: elastic scattering, where the trion remains bound, capturing scattering, where the heavy-hole exciton captures a free electron to form a trion, and dissociating scattering where the trion dissociates into a heavy-hole exciton and an extra free electron. The latter process is important due to the small binding energy of the trion with respect to the exciton. We 
neglect the scattering in which both electrons become unbound. In order to facilitate the matrix elements calculation we consider a simple two parameter Chandrasekhar type 22 trial function for the trion

$$
\begin{gathered}
\phi^{\operatorname{tr}}\left(r_{1 \mathrm{~h}}, r_{2 \mathrm{~h}}, z_{1}, z_{2}, z_{h}\right)=\frac{1}{A} \mathcal{N}_{\operatorname{tr}} \chi_{e}\left(z_{1}\right) \chi_{e}\left(z_{2}\right) \times \\
\chi_{h}\left(z_{h}\right)\left[\phi\left(r_{1 \mathrm{~h}}\right) \phi^{\prime}\left(r_{2 \mathrm{~h}}\right) \pm \phi^{\prime}\left(r_{1 \mathrm{~h}}\right) \phi\left(r_{2 \mathrm{~h}}\right)\right],
\end{gathered}
$$

where the $+(-)$ sign applies to the singlet (triplet) spin configuration. In Eq. (III.1), $r_{\mathrm{ih}}=\left|\mathbf{r}_{\mathrm{i}}-\mathbf{r}_{\mathrm{h}}\right|, \quad(i=1,2)$ are the in-plane coordinates of the two electrons with respect to the hole in-plane coordinate, $z_{i}$ are the electrons $z$ coordinates, and $\chi_{e}\left(z_{i}\right), \chi_{h}\left(z_{h}\right)$ are the confinement functions in the $z$ direction, taken to be the same for all constituents (Eq. (A-3)). The two electron orbitals are given by the normalized functions

$$
\begin{aligned}
\phi(r) & =\sqrt{\frac{2}{\pi \lambda^{2}}} \mathrm{e}^{-r / \lambda} \\
\phi^{\prime}(r) & =\sqrt{\frac{2}{\pi \lambda^{\prime 2}}} \mathrm{e}^{-r / \lambda^{\prime}},
\end{aligned}
$$

and the trion wavefunction normalization factor is given by

$$
\mathcal{N}_{\text {tr }}=\left(\frac{2}{L}\right)^{3 / 2} \frac{1}{\sqrt{1 \pm \kappa^{2}}}
$$

where

$$
\kappa \equiv\left\langle\phi \mid \phi^{\prime}\right\rangle=\frac{4 \lambda \lambda^{\prime}}{\left(\lambda+\lambda^{\prime}\right)^{2}} .
$$

The trial function (III.1) was used in 23 for the case of a two dimensional negative-donor center (i.e., $m_{h} \rightarrow \infty$ limit). In addition, these authors performed the binding energy calculations using a more complicated version of (III.1) which includes a correlation term (with an additional variational parameter). The variational parameters $\lambda, \lambda^{\prime}$ are calculated by maximizing the trion binding energy for the two spin configurations. The details of the binding energy calculation are given in Appendix C, resulting in $E_{b}^{\mathrm{tr}}=0.985 \mathrm{meV}, \lambda=143 \AA$, $\lambda^{\prime}=300 \AA\left(E_{b}^{\mathrm{tr}}=0.765 \mu \mathrm{eV}, \lambda=151 \AA, \lambda^{\prime}=7650 \AA\right)$ for the singlet (triplet) spin configuration, where $E_{b}^{\mathrm{tr}}$ is calculated with respect to the heavy hole $1 \mathrm{~S}$ neutral exciton energy. We note that the triplet configuration is barely bound, in accordance with the experimental observations, thus it can be disregarded. Comparing with the much more elaborate treatment of Stèbè et a13, our results are fairly accurate, giving us the confidence to proceed in calculating the trion-electron scattering matrix elements using the wave function (III.1).

We construct the state of a single trion with an inplane CM momentum $\mathbf{k}_{t}$, similarly to that of a single exciton (II.1):

$$
\begin{aligned}
\left|\mathbf{k}_{\mathbf{t}}\right\rangle= & \sum_{\substack{\mathbf{k}_{1}, \mathbf{k}_{2} \\
s_{1}, s_{2}}} \int d z_{1} d z_{2} d z_{h} \phi_{\alpha_{t} \mathbf{k}_{\mathrm{t}}+\mathbf{k}_{1}, \alpha_{t} \mathbf{k}_{\mathrm{t}}+\mathbf{k}_{2}}^{\mathrm{tr} *}\left(z_{1}, z_{2}, z_{h}\right) \times \\
& \xi_{S}^{*}\left(s_{1}, s_{2}\right) c_{-\mathbf{k}_{1}, z_{1}}^{s_{1} \dagger} c_{-\mathbf{k}_{2}, z_{2}}^{s_{2} \dagger} d_{\mathbf{k}_{\mathrm{t}}+\mathbf{k}_{1}+\mathbf{k}_{2}, z_{h}}^{\dagger}|0\rangle, \quad \text { (III.5) }
\end{aligned}
$$

where we have added the spin index to the electron creation operators, and denoted $\alpha_{t}=m_{\mathrm{e}} / M_{\mathrm{tr}}$, and $\xi_{S}\left(s_{1}, s_{2}\right)=\left\langle S \mid s_{1}, s_{2}\right\rangle$ as the projection of a generic spin configuration of two electrons on the singlet spin configuration. The in-plane Fourier transformed trion wave function in Eq. (III.5) is given by

$$
\phi_{\mathbf{k}_{1}, \mathbf{k}_{2}}^{\mathrm{tr}}\left(z_{1}, z_{2}, z_{h}\right)=\mathcal{N}_{\mathrm{tr}} \chi_{e}\left(z_{1}\right) \chi_{e}\left(z_{2}\right) \chi_{h}\left(z_{h}\right) \phi_{\mathbf{k}_{1}} \phi_{\mathbf{k}_{2}}^{\prime},
$$

where,

$$
\begin{aligned}
& \phi_{\mathbf{k}}=\sqrt{\frac{8 \pi \lambda^{2}}{A}} \frac{1}{\left[1+(\lambda k)^{2}\right]^{3 / 2}} \\
& \phi_{\mathbf{k}}^{\prime}=\sqrt{\frac{8 \pi \lambda^{\prime 2}}{A}} \frac{1}{\left[1+\left(\lambda^{\prime} k\right)^{2}\right]^{3 / 2}} .
\end{aligned}
$$

A state comprising a trion and a free electron having $\mathbf{k}_{\mathrm{e}}$, $z_{e}$, and $s_{e}$ will be written as

$$
\begin{gathered}
\left|\mathbf{k}_{\mathrm{t}} ; \mathbf{k}_{\mathrm{e}}\right\rangle=\sum_{\substack{\mathbf{k}_{1}, \mathbf{k}_{2} \\
s_{1}, s_{2}, s_{e}}} \int d z_{1} d z_{2} d z_{h} d z_{e} \xi_{S}^{*}\left(s_{1}, s_{2}\right) \times \\
\phi_{\alpha_{t} \mathbf{k}_{\mathrm{t}}+\mathbf{k}_{1}, \alpha_{t} \mathbf{k}_{\mathrm{t}}+\mathbf{k}_{2}}^{\mathrm{tr}}\left(z_{1}, z_{2}, z_{h}\right) \psi_{\mathbf{k}_{\mathrm{e}}}^{*}\left(z_{e}\right) \times \\
c_{-\mathbf{k}_{1}, z_{1}}^{s_{1} \dagger} c_{-\mathbf{k}_{2}, z_{2}}^{s_{2} \dagger} d_{\mathbf{k}_{\mathrm{t}}+\mathbf{k}_{1}+\mathbf{k}_{2}, z_{h}}^{\dagger} c_{\mathbf{k}_{e}, z_{e}}^{s_{e} \dagger}|0\rangle .
\end{gathered}
$$

In the following we shall use these wave functions to calculate the scattering matrix elements for the various scattering processes.

\section{A. Elastic scattering}

Applying the Coulomb interaction operators $V_{e 1}, V_{e 2}$, and $V_{e h}$ to the initial state of the system (III.8), and discarding the self energy contributions, we can write a generic expression for the three scattering matrix elements: 


$$
\begin{gathered}
\left\langle\mathbf{k}_{\mathbf{t}}+\mathbf{q} ; \mathbf{k}_{\mathrm{e}}-\mathbf{q}\left|V_{e i}\right| \mathbf{k}_{\mathbf{t}} ; \mathbf{k}_{\mathrm{e}}\right\rangle=\frac{1}{2} \sum_{\substack{\mathbf{k}_{1}, \mathbf{k}_{2} \\
\mathbf{k}_{1}^{\prime}, \mathbf{k}_{2}^{\prime}, \mathbf{q}_{1}}} \sum_{\substack{s_{1}, s_{2}, s_{2} \\
s_{1}^{\prime}, s_{e}^{\prime}}} \int d z_{i} d z_{i}^{\prime} d z_{h} d z_{h}^{\prime} V_{\mathbf{q}_{1}}\left(z_{e}-z_{i}\right) \phi_{\alpha_{t}}^{\mathrm{tr}}\left(\mathbf{k}_{\mathrm{t}}+\mathbf{q}\right)+\mathbf{k}_{1}^{\prime}, \alpha_{t}\left(\mathbf{k}_{\mathbf{t}}+\mathbf{q}\right)+\mathbf{k}_{2}^{\prime}\left(z_{i}^{\prime}\right) \times \\
\xi_{S}\left(s_{1}^{\prime}, s_{2}^{\prime}\right) \psi_{\mathbf{k}_{\mathrm{e}}-\mathbf{q}}\left(z_{e}^{\prime}\right) \phi_{\alpha_{t} \mathbf{k}_{\mathrm{t}}+\mathbf{k}_{1}, \alpha_{t} \mathbf{k}_{\mathrm{t}}+\mathbf{k}_{2}}^{\mathrm{tr} *}\left(z_{i}\right) \xi_{S}^{*}\left(s_{1}, s_{2}\right) \psi_{\mathbf{k}_{\mathrm{e}}}^{*}\left(z_{e}\right)\left\langle 0\left|c_{1} c_{2} c_{3} c_{4}^{\dagger} c_{5}^{\dagger} c_{6}^{\dagger}\right| 0\right\rangle, \quad(i=1,2, h),
\end{gathered}
$$

where we put the $1 / 2$ prefactor to indicate averaging over the initial electron spin states, and used a numeric index for the electron operators, indicating both spatial and spin degrees of freedom. Note that we have omitted the hole operators since they anticommute with the electron operators and do not contribute additional constraints. Using the electrons anticommutation relations, the Fermi vacuum expectation value of the operators reads:

$$
\begin{aligned}
\left\langle 0\left|c_{1} c_{2} c_{3} c_{4}^{\dagger} c_{5}^{\dagger} c_{6}^{\dagger}\right| 0\right\rangle & =\delta_{34}\left(\delta_{25} \delta_{16}-\delta_{15} \delta_{26}\right)+ \\
\delta_{24}\left(\delta_{15} \delta_{36}-\delta_{35} \delta_{16}\right) & +\delta_{14}\left(\delta_{35} \delta_{26}-\delta_{25} \delta_{36}\right),(\mathrm{I})
\end{aligned}
$$

where we have symbolically written $\delta_{i j}=\delta_{\mathbf{k}_{i}, \mathbf{k}_{j}} \delta\left(z_{i}-\right.$ $\left.z_{j}\right) \delta_{s_{i}, s_{j}}$. For the 1st electron interaction term we have:

$$
\begin{array}{ll}
1=\left(\mathbf{k}_{e}-\mathbf{q} ; z_{e}^{\prime} ; s_{e}^{\prime}\right) & 2=\left(-\mathbf{k}_{2}^{\prime} ; z_{2}^{\prime} ; s_{2}^{\prime}\right) \\
3=\left(-\mathbf{k}_{1}^{\prime} ; z_{1}^{\prime} ; s_{1}^{\prime}\right) & 4=\left(-\mathbf{k}_{1}-\mathbf{q}_{1} ; z_{1} ; s_{1}\right) \\
5=\left(-\mathbf{k}_{2} ; z_{2} ; s_{2}\right) & 6=\left(-\mathbf{k}_{e}+\mathbf{q}_{1} ; z_{e} ; s_{e}\right)
\end{array}
$$

The second electron interaction term is found by making the substitutions: $\mathbf{k}_{1} \rightarrow \mathbf{k}_{1}-\mathbf{q}_{1}, \mathbf{k}_{2} \rightarrow \mathbf{k}_{2}+\mathbf{q}_{1}$ in Eqs. (III.11). Similarly, the electron-hole interaction term is found by substituting: $\mathbf{k}_{1} \rightarrow \mathbf{k}_{1}-\mathbf{q}_{1}$ in (III.11). Applying the delta functions of (III.10) to Eq. (III.9), it is evident that the contributions of the $z$-dependent parts are identical for all three terms, since we have taken identical confinement functions for the electron and the hole (see Eq. A-3). Assuming that the trion remains in the singlet electron spin configuration, we sum over the spin degrees of freedom, resulting in selection rules for the three electron final spin states, which determine the relevant signs of the various terms. Performing the in-plane momentum integrations, and collecting the matrix elements into one direct and two exchange contributions, we finally find:

$$
\begin{aligned}
V_{\mathrm{dir}}(q)= & \mathcal{N}_{\mathrm{es}} h(\lambda q)\left\{\left[g\left(\lambda \alpha_{t} q / 2\right) g\left(\lambda^{\prime} \beta_{t} q / 2\right)+g\left(\lambda^{\prime} \alpha_{t} q / 2\right) g\left(\lambda \beta_{t} q / 2\right)+2 \kappa^{2} g\left(\tilde{\lambda} \alpha_{t} q\right) g\left(\tilde{\lambda} \beta_{t} q\right)\right]-\right. \\
& {\left.\left[g\left(\lambda \alpha_{t} q / 2\right) g\left(\lambda^{\prime} \alpha_{t} q / 2\right)+\kappa^{2} g^{2}\left(\tilde{\lambda} \alpha_{t} q\right)\right]\right\} }
\end{aligned}
$$

and

$$
\begin{aligned}
V_{\mathrm{exc}}^{(1)}\left(\mathbf{q}, \Delta \mathbf{k}_{t}\right)= & -\frac{\mathcal{N}_{\mathrm{es}}}{\pi} \int d^{2} k_{1} h\left(\lambda k_{1}\right)\left\{\left[\lambda \lambda^{\prime} \kappa g\left(\tilde{\lambda} \alpha_{t} q\right) g\left(\lambda\left|\mathbf{k}_{1}-\Delta \mathbf{k}_{t}+\alpha_{t} \mathbf{q}\right|\right)+\lambda^{\prime 2} g\left(\lambda \alpha_{t} q / 2\right) g\left(\lambda^{\prime}\left|\mathbf{k}_{1}-\Delta \mathbf{k}_{t}+\alpha_{t} \mathbf{q}\right|\right)\right] \times\right. \\
& {\left.\left[g\left(\lambda^{\prime}\left|\mathbf{k}_{1}-\Delta \mathbf{k}_{t}+\mathbf{q}\right|\right)-g\left(\lambda^{\prime}\left|\Delta \mathbf{k}_{t}-\mathbf{q}\right|\right)\right]+\left[\lambda \leftrightarrow \lambda^{\prime}\right] \times\left[\lambda \leftrightarrow \lambda^{\prime}\right]\right\} } \\
V_{\mathrm{exc}}^{(2)}\left(\mathbf{q}, \Delta \mathbf{k}_{t}\right)= & -\frac{\mathcal{N}_{\mathrm{es}}}{\pi} \int d^{2} k_{1} h\left(\lambda k_{1}\right)\left\{\left[\lambda \lambda^{\prime} \kappa g\left(\tilde{\lambda}\left|\mathbf{k}_{1}-\alpha_{t} q\right|\right) g\left(\lambda^{\prime}\left|\mathbf{q}-\Delta \mathbf{k}_{t}\right|\right)+\lambda^{2} g\left(\lambda^{\prime}\left|\mathbf{k}_{1}-\alpha_{t} \mathbf{q}\right| / 2\right) g\left(\lambda\left|\mathbf{q}-\Delta \mathbf{k}_{t}\right|\right)\right] \times\right. \\
& \left.g\left(\lambda\left|\mathbf{k}_{1}-\Delta \mathbf{k}_{t}+\alpha_{t} \mathbf{q}\right|\right)+\left[\lambda \leftrightarrow \lambda^{\prime}\right] \times\left[\lambda \leftrightarrow \lambda^{\prime}\right]\right\}
\end{aligned}
$$

where we have denoted $\beta_{t}=1-\alpha_{t}, \Delta \mathbf{k}_{t}=\mathbf{k}_{e}-\alpha_{t} \mathbf{k}_{t}$, and $\tilde{\lambda}=\lambda \lambda^{\prime} /\left(\lambda+\lambda^{\prime}\right)^{2}$. In Eqs. (III.12)-(III.13), the prefactor, multiplying the dimensionless quantities, is given by

$$
\mathcal{N}_{\mathrm{es}}=\frac{1}{1+\kappa^{2}} \frac{4 \pi e^{2} \lambda^{3}}{L^{2} A \epsilon_{0}} .
$$

We note that the direct term (III.12) diverges at $q \rightarrow 0$. This divergence originates from the infinite range of the Coulomb potential, which is manifest whenever a scattering event between two charged particles occurs (note that for the neutral exciton this divergence cancelled out, due to the equal contributions with opposite signs from the electron and hole constituting the exciton). In practice the Coulomb potential is screened by the presence of the 2DEG. Since we are considering a low density 2DEG, its Fermi wavevector, $k_{\mathrm{f}}=\sqrt{2 \pi n_{\mathrm{e}}}$ is small, thus the semiclassical (Thomas-Fermi) approximation, which assumes that $q \ll k_{\mathrm{f}}$, is inadequate 4 . In particular, the two-dimensional semiclassical analysis results $($ at $T=0)$ in a density-independent screening wavevector $q_{0}$, implying that the presence of a single electron is sufficient to screen the external potential, which is clearly unrealistic. 
We adopt instead the Lindhard approach which utilizes a perturbative scheme to evaluate the induced charge density in first order of the total potential (By total we mean the sum of the external potential, i.e., that of the trion, and the 2DEG potential that is induced by the trion's presence). The in-plane Fourier transformed dielectric function which results from the effect of the screening is given by 24

$$
\epsilon_{s}(q)=1+\frac{q_{0}}{q} g_{s}(q) \alpha_{s}(q)
$$

where

$$
q_{0}=\frac{2 m_{e} e^{2}}{\epsilon_{0} \hbar^{2}}=\frac{2}{a_{\mathrm{B}}}
$$

and $a_{\mathrm{B}}$ is the electrons effective bulk Bohr radius. Two factors appear in Eq. (III.15), which influence the screening. The first is the screening form factor given by

$$
g_{s}(q)=\int d z d z^{\prime} \chi^{2}(z) \chi^{2}\left(z^{\prime}\right) \mathrm{e}^{-q\left|z-z^{\prime}\right|}
$$

which arises from the finite $\mathrm{QW}$ width. In the infinite barrier limit we are considering, the confinement functions, $\chi(z)$, are given by Eq. (A-3), and $g_{s}(q)$ can be calculated analytically, resulting in

$$
g_{s}(q)=\frac{2}{(q L)^{2}} \frac{\mathrm{e}^{-L q}-1+L q+\frac{5}{8 \pi^{2}}(L q)^{3}+\frac{3}{32 \pi^{4}}(L q)^{5}}{\left[1+\left(\frac{L q}{2 \pi}\right)^{2}\right]^{2}}
$$

This screening form factor is smaller than 1, thus it reduces the effect of the screening, compared with the strictly two-dimensional case $\left(g_{s}(L \rightarrow 0)=1\right)$. The second factor which appears in Eq. (III.15) is given by

$$
\alpha_{s}(q)=\frac{1}{\pi} \int d^{2} k \frac{f_{\mathrm{fd}}(\mathbf{k}+\mathbf{q})-f_{\mathrm{fd}}(k)}{(\mathbf{k}+\mathbf{q})^{2}-k^{2}} .
$$

We note that $\alpha_{s}(q)$ does not depend on the particular details of the QW, but only on the two-dimensional nature of the 2DEG motion. Substituting the screening dielectric function into the Coulomb interaction, we find the screened potential to be

$$
V_{q}^{s}(z)=\frac{1}{\epsilon_{s}(q)} \frac{2 \pi e^{2}}{A \epsilon_{0}} \frac{e^{-|z| q}}{q}=\frac{2 \pi e^{2}}{A \epsilon_{0}} \frac{e^{-|z| q}}{q+q_{0} g_{s}(q) \alpha_{s}(q)} ;
$$

thus the use of the screened potential implies replacing $h(\lambda q)$ in Eq. (III.12) with

$$
h_{s}(\lambda q)=\frac{h(\lambda q)}{1+\frac{4 \lambda^{3}}{L^{2} a_{\mathrm{B}}} h(\lambda q) \alpha_{s}(q)} .
$$

In general, the effect of the quasi two-dimensional screening saturates in larger electron densities, in contradistinction to the bulk case, resulting in a complicated dependence of the trion linewidth on $n_{\mathrm{e}}$. We note that since there are no divergences in the exchange terms, replacing $h\left(\lambda k_{1}\right)$ with $h_{s}\left(\lambda k_{1}\right)$ in Eqs. (III.13) amounts to a very small effect (less than $1 \%$ in the relevant parameter range).
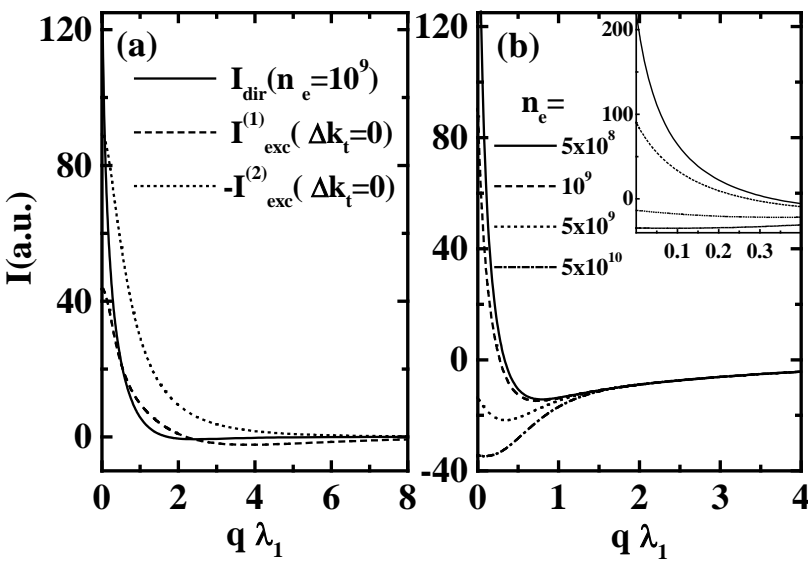

FIG. 5: Calculated dimensionless matrix elements at $T=$ $5 K$ for the case of $\Delta \mathbf{k}_{\mathrm{t}}=0$, vs. transferred momentum: (a) Direct $\left(n_{\mathrm{e}}=10^{9} \mathrm{~cm}^{-2}\right)$ and exchange terms (note that since the exchange terms are not affected by the screening, their dependence on $n_{\mathrm{e}}$ is negligible) ; (b) Total matrix element for various electron densities $n_{\mathrm{e}}$. The inset provides a close-up at small q.

Figure 5a shows the direct and exchange dimensionless matrix elements as a function of transferred momentum $q$, for the case $\Delta \mathbf{k}_{\mathrm{t}}=0$, where the angular dependence of $V_{\text {exc }}^{(1)}, V_{\text {exc }}^{(2)}$ disappears. The effect of the screening on the total dimensionless matrix element is shown in Fig. 5b, where various electron densities are considered, giving rise to a change in the screening action. As expected, the effect of the screening is noticeable in the region of small momenta of the direct matrix element.

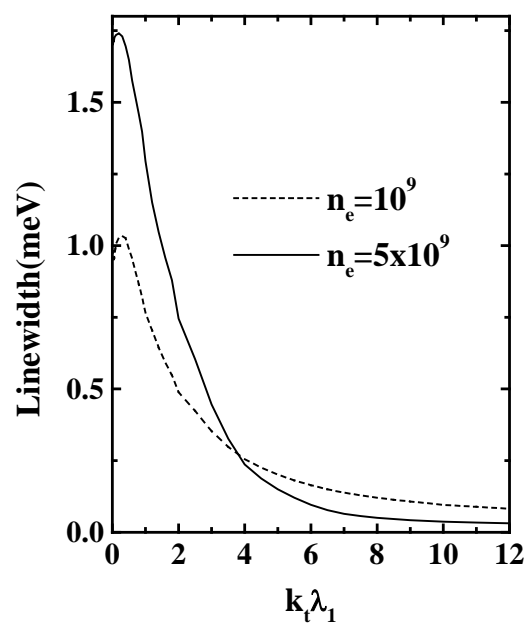

FIG. 6: Calculated trion linewidth due to elastic scattering vs. initial trion momentum, at $T=5 \mathrm{~K}$ for two electron densities. 
The elastic scattering rate of the trion, $w_{\mathrm{t}-\mathrm{e}}\left(\mathbf{k}_{\mathrm{t}} \rightarrow\right.$ $\mathbf{k}_{\mathrm{t}}+\mathbf{q}$ ), is calculated by writing the energy-momentum conservation equation and finding its solution $k_{\mathrm{e}}^{*}$, similarly to Eqs. (II.9)-(II.11). Integrating over all final trionic states, we calculate the trion linewidth due to elastic electron scattering, $\Gamma_{\mathrm{t}-\mathrm{e}}\left(k_{\mathrm{t}}\right)$, plotted for two electron densities in Fig. 6. The linewidth dependence on $n_{\mathrm{e}}$ is quite complicated. First, as we are considering $T=5 \mathrm{~K}$, the density range for which the linewidth exhibits a linear dependence on $n_{\mathrm{e}}$ is quite small, similar to the neutral exciton scattering (cf. Fig. 2 $2 \mathrm{c}$ ). Second, the screening potential becomes larger as the density decreases, thus for very small densities $\left(n_{\mathrm{e}} \lesssim 5 \cdot 10^{8}\right)$ decreasing $n_{\mathrm{e}}$ results in a larger trion linewidth. We note that for such low densities, it is probable that other screening mechanisms become appreciable (e.g. lattice impurities), thus such a behavior will probably not be observed experimentally.

\section{B. Electron capture}

We now consider the case in which a heavy-hole exciton captures a free electron thereby forming a trion. The initial and final states of the system are given by Eqs. (II.2) and (III.5), respectively. The calculation of the Coulomb interaction matrix elements between these states essentially follows the procedure described in the previous section. In this process there is no exchange term. Instead, the two electrons which constitute the trion form a singlet spin configuration. Defining $\Delta \mathbf{k} \equiv \alpha_{\mathrm{t}} \mathbf{k}_{\mathrm{t}}-\alpha_{\mathrm{x}} \mathbf{k}_{\mathrm{x}}$, the calculation yields:

$$
\begin{aligned}
& V_{\text {cap }}(\Delta k)=\mathcal{N}_{\text {cap }} \int d^{2} k_{1} h\left(\lambda_{0} k_{1}\right)\left\{\frac{\lambda \lambda^{\prime}}{\left(\lambda_{0}+\lambda^{\prime}\right)^{2}} \times\right. \\
& {\left[g\left(\tilde{\lambda}^{\prime}\left|\mathbf{k}_{1}+\Delta \mathbf{k}\right|\right)-g\left(\tilde{\lambda}^{\prime} \Delta k\right)\right] g\left(\lambda\left|\mathbf{k}_{1}+\frac{\Delta \mathbf{k}}{\alpha_{\mathrm{x}}}\right|\right)+} \\
& \frac{\lambda \lambda^{\prime}}{\left(\lambda_{0}+\lambda\right)^{2}}\left[g\left(\tilde{\lambda}\left|\mathbf{k}_{1}+\Delta \mathbf{k}\right|\right)-g(\tilde{\lambda} \Delta k)\right] \times \quad(\text { III } .22 \\
& \left.g\left(\lambda^{\prime}\left|\mathbf{k}_{1}+\frac{\Delta \mathbf{k}}{\alpha_{\mathrm{x}}}\right|\right)\right\}
\end{aligned}
$$

where we have defined the reduced Bohr radii: $\tilde{\lambda}=$ $\lambda_{0} \lambda /\left(\lambda_{0}+\lambda\right)$ and $\tilde{\lambda}^{\prime}=\lambda_{0} \lambda^{\prime} /\left(\lambda_{0}+\lambda^{\prime}\right)$, and the prefactor:

$$
\mathcal{N}_{\text {cap }}=\frac{8 e^{2} \lambda_{0}^{4}}{\sqrt{1+\kappa^{2}} \sqrt{\pi A} L^{2} \epsilon_{0}} .
$$

Fig. Fa shows the dependence of the dimensionless matrix element on $\Delta k$. Proceeding as before, we write conservation of energy:

$$
E_{\mathrm{x}}\left(k_{\mathrm{x}}\right)+E_{\mathrm{e}}\left(\mathbf{k}_{\mathrm{t}}-\mathbf{k}_{\mathrm{x}}\right)=E_{\mathrm{t}}\left(\mathrm{k}_{\mathrm{t}}\right)+E_{b}^{\mathrm{tr}}
$$

which is satisfied for

$$
k_{\mathrm{t}}^{*}=\frac{1}{\beta_{t}}\left(k_{\mathrm{x}} \cos \theta \pm \sqrt{\beta_{t} \frac{E_{b}^{\mathrm{tr}} m_{\mathrm{e}}}{\hbar^{2}}-k_{\mathrm{x}}^{2} \sin ^{2} \theta}\right)
$$

where $\theta=\angle\left(\mathbf{k}_{\mathrm{x}}, \mathbf{k}_{\mathrm{t}}\right)$. We note that for the energy conserving solutions, $k_{\mathrm{t}}^{*}$, the interaction matrix element is constant since $\Delta \mathbf{k}\left(k_{\mathrm{t}}^{*}\right)=\sqrt{\alpha_{x} \alpha_{t} E_{b}^{\mathrm{tr}} m_{\mathrm{e}} / \hbar^{2}}$. Thus, the dependence of the heavy hole exciton linewidth on its initial momentum, shown in Fig. $7 \mathrm{~b}$, comes solely from energy momentum conservation and the Fermi-Dirac distribution of the scattered electron: $f_{\mathrm{fd}}\left(\mathbf{k}_{\mathrm{e}}\right)=f_{\mathrm{fd}}\left(\mathbf{k}_{\mathrm{t}}^{*}-\mathbf{k}_{\mathrm{x}}\right)$.
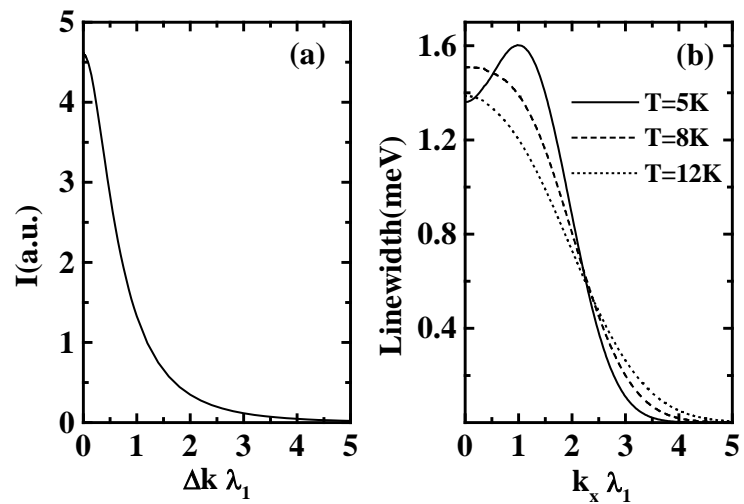

FIG. 7: (a) Calculated dimensionless matrix element of the capturing process vs. $\Delta k$ at $T=5 K$; (b) $X_{\mathrm{hh}}$ calculated linewidth due to capturing scattering for $n_{\mathrm{e}}=10^{9} \mathrm{~cm}^{-2}$ at three temperatures.

\section{Charged exciton dissociation}

Lastly, we consider the case in which the scattered trion dissociates into a neutral exciton and a free electron. This scattering process is shown schematically in Fig. 8 . The initial state of the system is given by Eq. (III.8) while the final state is that of three free particles, symbolically written as $\left|\mathbf{k}_{\mathrm{x}} ; \mathbf{k}_{\mathrm{e}} ; \mathbf{k}_{\mathrm{t}}+\mathbf{q}-\mathbf{k}_{\mathrm{x}}\right\rangle$, where $\mathbf{q}$ is the momentum transferred from the free electron to the trion, and $\mathbf{k}_{\mathrm{x}}$ is the neutral exciton CM momentum.

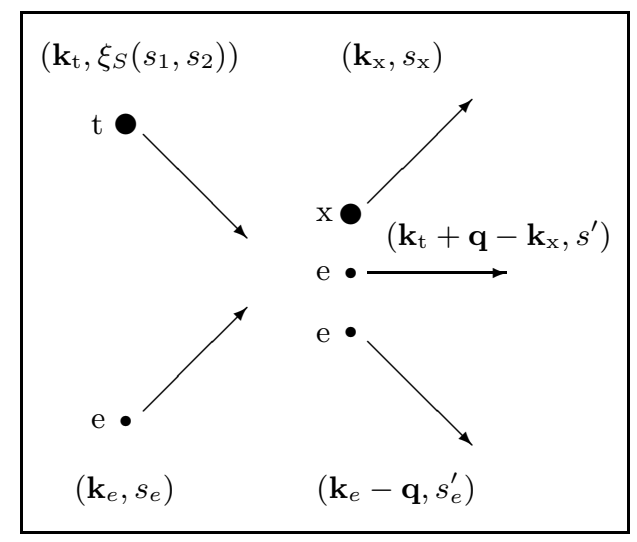

FIG. 8: Electron-trion inelastic scattering scheme

Proceeding as before, we calculate the interaction matrix elements between the initial and final states of the 
system. To this end we denote the final spin state of the three electrons as $\left|s_{\mathrm{x}}, s^{\prime}, s_{e}^{\prime}\right\rangle$ (see Fig. 8). Assuming the interaction is spin independent, this final state will be one of the two states: $(s, m)=\left(\frac{1}{2}, \pm \frac{1}{2}\right)$, where the state with $m=\frac{1}{2}\left(m=-\frac{1}{2}\right)$ corresponds to the initial electron spin $s_{e}$ being up (down). Actually, we need not concern ourselves with the exact form of the final electron spin state, since the projection of the initial spin state (which is a tensor product of a singlet state with the additional free electron spin) on a generic final spin state will extract the relevant contributions. The proper (anti)symmetrization of this final spin state is performed inherently by the anticommutation relations of the electron operators, as in Eq. (III.10). As in the elastic scattering case, we find one direct and two exchange contributions. Denoting $\Delta \mathbf{k}_{\mathrm{t}}=\mathbf{k}_{\mathrm{e}}-\alpha_{\mathrm{t}} \mathbf{k}_{\mathrm{t}}$, and $\Delta \mathbf{k}_{\mathrm{x}}=\mathbf{k}_{\mathrm{e}}-\alpha_{\mathrm{x}} \mathbf{k}_{\mathrm{x}}$ as before, these terms are expressed as:

$$
\begin{aligned}
V_{\text {dir }}(\Delta \mathbf{k}, \mathbf{q})= & \mathcal{N}_{\text {dis }} h_{s}\left(\lambda_{0} q\right)\left(\frac{\lambda \lambda^{\prime}}{\left(\lambda_{0}+\lambda\right)^{2}}\left\{g\left(\lambda^{\prime} \frac{\Delta k}{\alpha_{\mathrm{x}}}\right) g(\tilde{\lambda} \Delta k)+g\left(\lambda^{\prime}\left|\frac{\Delta \mathbf{k}}{\alpha_{\mathrm{x}}}+\mathbf{q}\right|\right)[g(\tilde{\lambda}|\Delta \mathbf{k}+\mathbf{q}|)-g(\tilde{\lambda} \Delta k)]\right\}+\right. \\
& \left.\frac{\lambda \lambda^{\prime}}{\left(\lambda_{0}+\lambda^{\prime}\right)^{2}}\left\{\begin{array}{l}
\lambda \leftrightarrow \lambda^{\prime} \\
\tilde{\lambda} \rightarrow \tilde{\lambda}^{\prime}
\end{array}\right\}\right)
\end{aligned}
$$

and

$$
\begin{aligned}
& V_{\mathrm{exc}}^{(1)}\left(\mathbf{q}, \Delta \mathbf{k}_{\mathrm{t}}, \Delta \mathbf{k}_{\mathrm{x}}\right)=-\frac{\lambda \lambda^{\prime}}{\pi} \mathcal{N}_{\mathrm{dis}} \int d^{2} k_{1} h\left(\lambda_{0} k_{1}\right) g\left(\lambda_{0}\left|\mathbf{k}_{1}-\Delta \mathbf{k}_{\mathrm{x}}\right|\right)\left(\left\{\left[g\left(\lambda\left|\mathbf{k}_{1}-\frac{\Delta \mathbf{k}}{\alpha_{\mathrm{x}}}-\mathbf{q}\right|\right)-g\left(\lambda\left|\frac{\Delta \mathbf{k}}{\alpha_{\mathrm{x}}}+\mathbf{q}\right|\right)\right] \times\right.\right. \\
& \left.\left.g\left(\lambda^{\prime}\left|\mathbf{q}-\Delta \mathbf{k}_{\mathrm{t}}\right|\right)+g\left(\lambda\left|\mathbf{k}_{1}-\Delta \mathbf{k}_{\mathrm{t}}+\mathbf{q}\right|\right) g\left(\lambda^{\prime}\left|\frac{\Delta \mathbf{k}}{\alpha_{\mathrm{x}}}+\mathbf{q}\right|\right)\right\}+\left\{\lambda \leftrightarrow \lambda^{\prime}\right\}\right) \\
& V_{\mathrm{exc}}^{(2)}\left(\mathbf{q}, \Delta \mathbf{k}_{\mathrm{t}}, \Delta \mathbf{k}_{\mathrm{x}}\right)=-\frac{1}{2} \mathcal{N}_{\text {dis }} h_{s}\left(\lambda_{0}\left|\frac{\Delta \mathbf{k}_{\mathrm{x}}}{\alpha_{\mathrm{x}}}-\frac{\Delta \mathbf{k}_{\mathrm{t}}}{\alpha_{\mathrm{t}}}+\mathbf{q}\right|\right)\left(\frac { \lambda \lambda ^ { \prime } } { ( \lambda _ { 0 } + \lambda ) ^ { 2 } } \left\{g\left(\lambda^{\prime}\left|\Delta \mathbf{k}_{\mathrm{t}}-\mathbf{q}\right|\right) g\left(\tilde{\lambda}\left|\frac{\Delta \mathbf{k}_{\mathrm{t}}-\beta_{\mathrm{x}} \Delta \mathbf{k}_{\mathrm{x}}}{\alpha_{\mathrm{x}}}-\mathbf{q}\right|\right)+\right.\right. \\
& \left.\left.g(\tilde{\lambda} \Delta k)\left[g\left(\lambda^{\prime} \frac{\Delta k}{\alpha_{\mathrm{x}}}\right)-g\left(\lambda^{\prime}\left|\Delta \mathbf{k}_{\mathrm{t}}-\mathbf{q}\right|\right)\right]\right\}+\frac{\lambda \lambda^{\prime}}{\left(\lambda_{0}+\lambda^{\prime}\right)^{2}}\left\{\begin{array}{l}
\lambda \leftrightarrow \lambda^{\prime} \\
\tilde{\lambda} \rightarrow \tilde{\lambda}^{\prime}
\end{array}\right\}\right),
\end{aligned}
$$

where $\tilde{\lambda}, \tilde{\lambda}^{\prime}$ and $\Delta k$ were defined in the previous subsection and the prefactor multiplying the dimensionless quantities in Eqs. ([II.26)-(III.27), is given by

$$
\mathcal{N}_{\text {dis }}=\frac{16 \sqrt{2} e^{2} \lambda_{0}^{4}}{\sqrt{1+\kappa^{2}} L^{2} \epsilon_{0}}\left(\frac{2 \pi}{A}\right)^{3 / 2}
$$

The direct term (III.26) and the 2nd exchange term (III.27b) diverge at $q \rightarrow 0$ and at $\left(\Delta \mathbf{k}_{\mathrm{x}} / \alpha_{\mathrm{x}}-\Delta \mathbf{k}_{\mathrm{t}} / \alpha_{\mathrm{t}}+\right.$ q) $\rightarrow 0$, respectively, implying the need of using a screened Coulomb potential, as was done in the trion elastic scattering. Note that the direct term (III.26) depends only on $k_{\mathrm{t}}, k_{\mathrm{x}}, q$, and on the angles $\gamma=\angle\left(\mathbf{k}_{\mathrm{t}}, \mathbf{q}\right)$ and $\nu=\angle\left(\mathbf{k}_{\mathrm{t}}, \mathbf{k}_{\mathrm{x}}\right)$, whereas the exchange terms depend also on the initial electron momentum $k_{\mathrm{e}}$ and on the angle $\delta=\angle\left(\mathbf{k}_{\mathrm{e}}, \mathbf{q}\right)$. In practice, we express everything in terms of $q, \Delta k_{\mathrm{t}}, \Delta k_{\mathrm{x}}$, and the angles: $\theta_{1}=\angle\left(\Delta \mathbf{k}_{\mathrm{t}}, \Delta \mathbf{k}_{\mathrm{x}}\right)$, $\theta_{2}=\angle\left(\Delta \mathbf{k}_{\mathbf{x}}, \mathbf{q}\right)$, and $\theta_{3}=\angle\left(\Delta \mathbf{k}_{\mathrm{t}}, \mathbf{q}\right)$, given by:

$$
\begin{aligned}
\cos \theta_{1}= & \frac{1}{\Delta k_{\mathrm{x}} \Delta k_{\mathrm{t}}}\left(k_{\mathrm{e}}^{2}-\alpha_{\mathrm{x}} k_{\mathrm{x}} k_{\mathrm{e}} \cos (\gamma-\delta-\nu)-\right. \\
& \left.\alpha_{\mathrm{t}} k_{\mathrm{t}} k_{\mathrm{e}} \cos (\gamma-\delta)+\alpha_{\mathrm{x}} \alpha_{\mathrm{t}} k_{\mathrm{x}} k_{\mathrm{t}} \cos \nu\right)(\text { III.29a) } \\
\cos \theta_{2}= & \frac{k_{\mathrm{e}} \cos \delta-\alpha_{\mathrm{x}} k_{\mathrm{x}} \cos (\gamma-\nu)}{\Delta k_{\mathrm{x}}} \\
\cos \theta_{3} & =\frac{k_{\mathrm{e}} \cos \delta-\alpha_{\mathrm{t}} k_{\mathrm{t}} \cos \gamma}{\Delta k_{\mathrm{t}}} .
\end{aligned}
$$

The calculation of the scattering rate is performed as before, where conservation of energy now reads:

$$
\begin{aligned}
E_{\mathrm{t}}\left(k_{\mathrm{t}}\right)+E_{\mathrm{e}}\left(k_{\mathrm{e}}\right)= & E_{b}^{\mathrm{tr}}+E_{\mathrm{x}}\left(k_{\mathrm{x}}\right)+E_{\mathrm{e}}\left(\mathbf{k}_{\mathrm{t}}+\mathbf{q}-\mathbf{k}_{\mathrm{x}}\right)+ \\
& E_{\mathrm{e}}\left(\mathbf{k}_{\mathrm{e}}-\mathbf{q}\right) .
\end{aligned}
$$

Equation (III.30) is satisfied for

$$
\begin{aligned}
k_{\mathrm{e}}^{*}= & \frac{1}{q \cos \delta}\left\{\frac{m_{e}}{\hbar^{2}} E_{b}^{\mathrm{tr}}+q^{2}+\frac{1}{2 \beta_{\mathrm{t}}}\left(\beta_{\mathrm{t}} \mathbf{k}_{\mathrm{t}}-\mathbf{k}_{\mathrm{x}}\right)^{2}+\right. \\
& \left.q\left[k_{\mathrm{t}} \cos \gamma-k_{\mathrm{x}} \cos (\gamma-\nu)\right]\right\} .
\end{aligned}
$$

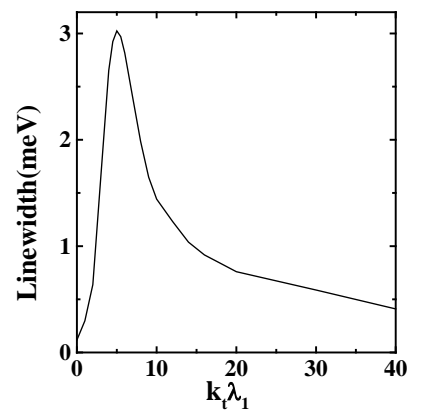

FIG. 9: Calculated trion linewidth due to inelastic scattering with electrons vs. its initial momentum, for $n_{\mathrm{e}}=10^{9} \mathrm{~cm}^{-2}$.

Using Fermi's golden rule as in Eq. (II.17), we find the scattering rate $w_{\mathrm{t}-\mathrm{e}}\left(\mathbf{k}_{\mathrm{t}} \rightarrow \mathbf{k}_{\mathrm{t}}+\mathbf{q}\right)$. Integrating over all 
final trionic states, we find the trion linewidth due to inelastic scattering as a function of its initial momentum $\mathbf{k}_{\mathrm{t}}, \Gamma_{\mathrm{t}-\mathrm{e}}^{\mathrm{dis}}\left(\mathbf{k}_{\mathrm{t}}\right)$, shown in Fig. 9 for $n_{\mathrm{e}}=10^{9} \mathrm{~cm}^{-2}$.

\section{EXCITONS AND TRIONS IN QW'S CONTAINING A 2DEG}

In this section we apply the linewidth calculations for the various scattering processes, presented in the previous sections, in order to calculate the line shapes of neutral excitons and trions. These line shapes can be directly compared with measured reflection spectra, taken from MTQW structures containing a variable density 2DEG. Without electrons in the QW and for $T \lesssim 100 K$, the two dominant scattering processes are due to acoustic phonons and static disorder. We note that the radiative lifetime of a free $\mathrm{QW}$ exciton is $\tau_{\text {rad }} \approx 25 \mathrm{ps}$, resulting in a radiative homogeneous broadening of $\gamma_{\mathrm{rad}} \approx 26 \mu \mathrm{eV}$. The exciton - acoustic phonon interaction leads to a homogeneous broadening whose Lorentzian line shape, $\mathcal{L}_{0}$, is characterized by $\gamma_{\mathrm{phon}}=0.2 \mathrm{meV}$ for the $X_{\mathrm{hh}}$ and $\gamma_{\text {phon }}=0.28 \mathrm{meV}$ for the $X_{\mathrm{lh}}$ at $T=80 K$, using the parameters of GaAs/AlAs QW's25. The 1S exciton energy, $E_{1 \mathrm{~S}}$, has a Gaussian distribution (characterized by $\left.\gamma_{\text {dis }}\right)$ due to the roughness of the QW surface. Convolving these two distributions results in the familiar Voigt functione 26

$$
\mathcal{I}_{0}(E)=\frac{\gamma_{\mathrm{phon}}}{(2 \pi)^{3 / 2} \gamma_{\mathrm{dis}}} \int_{-\infty}^{\infty} \frac{\mathrm{e}^{-E_{0}^{2} / 2 \gamma_{\mathrm{dis}}^{2}}}{\left(E-E_{0}\right)^{2}+\left(\gamma_{\mathrm{phon}} / 2\right)^{2}} d E_{0}
$$

where we take $E_{1 \mathrm{~S}}=0$. When a low density $2 \mathrm{DEG}$ is present, $\mathcal{I}_{0}(E)$ is convolved with a Lorentzian lineshape that is associated with the homogeneous broadening that is due to the electron scattering:

$$
\mathcal{I}(E)=\int_{0}^{\infty} \mathcal{I}_{0}\left(E-E^{\prime}\right) \mathcal{L}_{\mathrm{x}-\mathrm{e}}\left(E^{\prime}, \Gamma_{\mathrm{x}-\mathrm{e}}\left(E^{\prime}\right)\right) d E^{\prime},
$$

where $\Gamma_{\mathrm{x}-\mathrm{e}}\left(E^{\prime}\right)$ is given by Eq. (II.12), with the exciton energy, $E^{\prime}$, corresponding to $k_{\mathrm{x}}$. A pictorial description of the convolution in Eq. (IV.2) is given in Fig. 10, for both elastic and dissociating exciton-electron scattering processes (the trion-electron scattering exhibits a similar behavior). It is seen that the electron scattering admixes excitons having $k_{\mathrm{x}}>0$ with the $k_{\mathrm{x}}=0$ state. The degree of admixture is determined by the value of the Lorentzian that is due to electron scattering, given in the figure by the dashed line. Note that since $\Gamma_{\mathrm{x}-\mathrm{e}}\left(E^{\prime}\right)$ is a decreasing function of $E^{\prime}$, the Lorentzian peak is shifted to higher exciton energies. (This is observed experimentally as an increased shift with increasing $n_{e}$ ). In the calculation, the Gaussian width, associated with the inhomogeneity, was taken as a fitting parameter, yielding the value $\sigma_{\text {dis }}=$ $0.2 \mathrm{meV}$ for both $X_{\mathrm{hh}}$ and $X_{\mathrm{lh}}$ (the same value was taken also for the trion line).

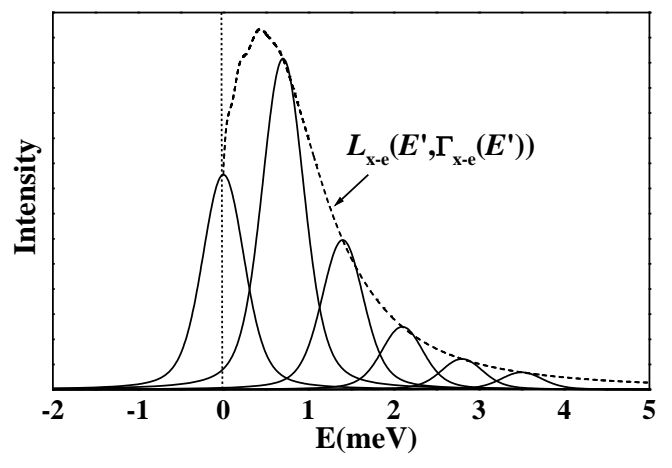

FIG. 10: A schematic picture of the convolution resulting from electron scattering. The solid lines represent various exciton initial line shapes $\mathcal{I}_{0}\left(E-E^{\prime}\right)$. The contributions of states with $k_{\mathrm{x}}>0$ to the $k_{\mathrm{x}}=0$ state are weighted by the value of the Lorentzian $\mathcal{L}_{\mathrm{x}-\mathrm{e}}\left(E^{\prime}, \Gamma_{\mathrm{x}-\mathrm{e}}\left(E^{\prime}\right)\right)$, given by the dashed line.

The calculated line shapes are identified with the imaginary part of the dielectric function in the $\mathrm{QW}$ that is related to the exciton (trion) resonance. Use of the Kramers-Kronig relations yields the real part of the dielectric function. Examples of calculated exciton line shapes together with the associated real part of the dielectric function are depicted in Fig 11 for two electron densities.
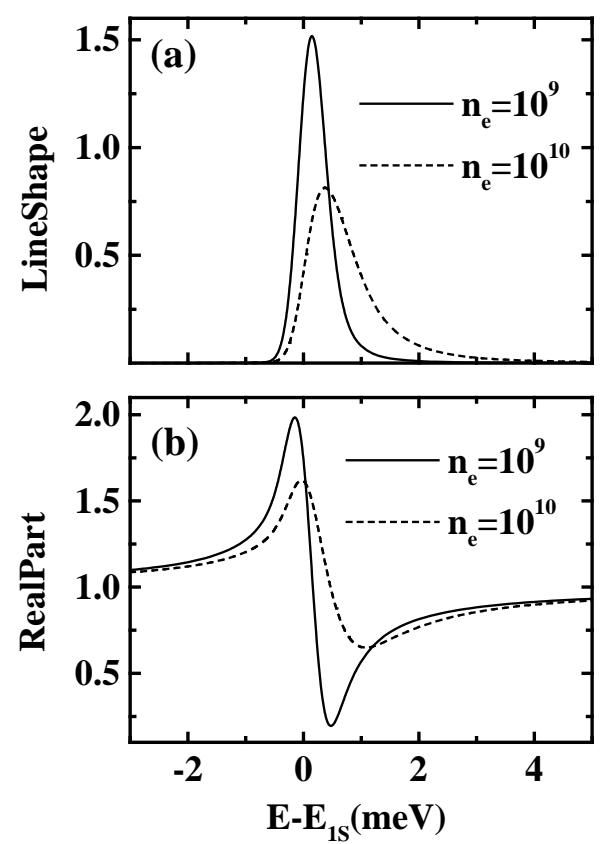

FIG. 11: (a) Heavy-hole $\left(M_{\mathrm{x}}=0.177 m_{e}\right)$ exciton normalized line shapes due to scattering with electrons for two electron densities; (b) The corresponding dielectric function real part.

Combining the three exciton line shapes (heavy-hole and light-hole neutral excitons, and heavy-hole trion), by 
using the appropriate oscillator strengths, the reflection spectra of a QW can be obtained. For high temperature $(T=80 K)$, where the trion is dissociated, these calculated spectra and their dependence on $n_{\mathrm{e}}$ were sh be in good agreement with the experimental datad.

In the low temperature regime $(2 K<T<20 K)$, the vindication of our results with experimental measurements is harder to perform. Since the heavy hole exciton and trion lines are very close in energy, and the trion oscillator strength is much lower than that of the exciton, it is practically impossible to discriminate between them by reflection measurements. PL experiments were performed in MTQW with 2DEG, at low temperatures, facilitating the observation of the trion line 17 , but there the detailed balance between the exciton and the trion line intensities is determined by the 2DEG density, and the system dynamics should be addressed by solving, e.g., rate equations.
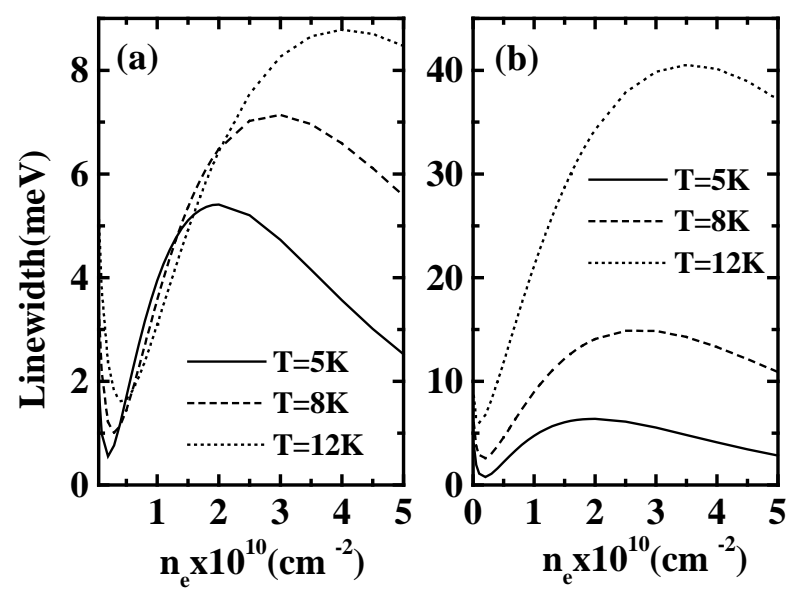

FIG. 12: Trion's linewidth vs. electron density, for three temperatures due to (a) elastic scattering ; (b) both elastic and dissociating scattering.

In Fig. $12 \mathrm{a}$ the trion linewidth due to elastic electron scattering is shown as a function of $n_{\mathrm{e}}$ for three temperatures. The combined effect of both elastic and dissociating electron scattering on the trion linewidth in shoyn in Fig. 12 b. Qualitatively, the trion line observed in 17 indeed broadens considerably at much lower $n_{\mathrm{e}}$ than the exciton line, as indicated by our results. The electron densities stated in 17 are probably an overestimate, as implied by the fact that the neutral exciton linewidth remains unchanged up to $n_{\mathrm{e}}=3 \cdot 10^{10} \mathrm{~cm}^{-2}$, in clear contradiction to both our calculations and other experimental data (e.g. J). It should be noted that calibrating the photoexcitation intensity $I_{L}$ to $n_{\mathrm{e}}$ in MTQWs is not an easy task. Even if one assumes a linear dependence of $n_{\mathrm{e}}$ on $I_{L}$ (which is valid for a limited range), the slope depends on the temperature, since the holes tunneling times decrease exponentially with $T$, so a different calibration should be performed not only for different experiments, but also for each temperature. Finally, the strong en- hancement of the dissociating scattering with increasing temperature, evident in Fig. 12b, implies that the trion line should practically vanish for $T>10 \mathrm{~K}$ at very low electron densities.

\section{SUMMARY AND CONCLUSIONS}

In this paper we presented a theoretical study of the various exciton-electron scattering processes that take place in a $\mathrm{QW}$ which contains a low density 2DEG. We have demonstrated that even for small densities, of the order of $n_{\mathrm{e}} \approx 5 \cdot 10^{9} \mathrm{~cm}^{-2}$ in the case of a GaAs/AlAs QW, exciton-electron scattering is much more efficient than both exciton-phonon and exciton-exciton scattering, thus playing a central role in the system dynamics. The dependence of the exciton linewidth on $n_{\mathrm{e}}$ was shown to be drastically different for the cases of a classical $(T=80 K)$ and a degenerate $(T=5 K)$ 2DEG. In general, the trion-electron scattering poses a much harder problem, one of the major difficulties being the evaluation of the screening effects. We showed that the screening model has a crucial effect on the trion-electron scattering matrix elements, and hence on the resulting trion linewidth. This is particularly true for very low densities $\left(n_{\mathrm{e}} \lesssim 5 \cdot 10^{8} \mathrm{~cm}^{-2}\right)$, where the 2DEG screening is less effective, making other possible mechanisms more dominant. Using the calculated scattering rates we showed that dissociating scattering can produce a sizable broadening of the excitonic lines, in particular for the trion, whose binding energy is considerably smaller than that of the neutral exciton.

A simple method to produce excitonic line shapes from the calculated linewidths was devised, allowing our results to be easily compared with experimental reflection spectra. A reliable source of experimental data is achievable from reflection measurements taken from a MTQW structure, embedded in a microcavity (MC). In the limit of large MC mode energy, the exciton linewidths are restored, since their coupling with the MC mode is barely noticeable. A major advantage of $\mathrm{MC}$ experiments is that the trion line is easily resolved by reflection 30 , allowing a direct comparison with theory.

The calculated interaction matrix elements can be readily used to evaluate polariton-electron scattering rates in MCs with 2DEG. In a recent experiment we have demonstrated polariton final-state stimulation, assisted by these scattering mechanisms, which was hitherto obseryed using polariton-polariton scattering mechanism 31 32. Incorporation of the various scattering processes which were considered in this paper, in a consistent explanation for these nonlinear effects, will be the topic of a forthcoming publication. 


\section{Acknowledgments}

This work was supported by the United States - Israel Binational Science Foundation (BSF), Jerusalem, Israel, by the Fund for Promotion of Research at the Technion, and by the Technion VPR Fund - Jewish Communities of Germany Research Fund. G.R. gratefully acknowledges the financial help from the Technion.

\section{Appendix A}

In this appendix we provide the details of the direct term calculation. Separating the coordinates in the QW plane $(x-y)$ from the perpendicular coordinate $(z)$, and denoting the electron and hole in-plane momenta by $\mathbf{k}_{1}$ and $\mathbf{k}_{2}$, respectively, we write the in-plane Fourier transform of the exciton wave function $\Phi\left[\left(\mathbf{r}_{e \|}, z_{e}\right),\left(\mathbf{r}_{h \|}, z_{h}\right)\right]$

$$
\begin{aligned}
\Phi_{\mathbf{k}_{1}, \mathbf{k}_{2}}\left(z_{e}, z_{h}\right)= & \frac{1}{A} \int d^{2} r_{e \|} d^{2} r_{h \|} \Phi\left[\left(\mathbf{r}_{e \|}, z_{e}\right),\left(\mathbf{r}_{h \|}, z_{h}\right)\right] \times \\
& \mathrm{e}^{-\mathrm{i}\left(\mathbf{k}_{1} \cdot \mathbf{r}_{e \|}+\mathbf{k}_{2} \cdot \mathbf{r}_{h \|}\right)}, \quad(\mathrm{A}-1)
\end{aligned}
$$

where $A$ denotes the QW surface area. Transforming to center-of-mass $(\mathrm{CM})$ and relative coordinates in the QW plane $\left(\mathbf{R}_{\|}=\alpha \mathbf{r}_{e \|}+\beta \mathbf{r}_{h \|}, \mathbf{r}_{\|}=\mathbf{r}_{e \|}-\mathbf{r}_{h \|}\right.$, where $\alpha=m_{\mathrm{e}} / M_{\mathrm{x}}, \beta=1-\alpha$, and $m_{\mathrm{e}}, M_{\mathrm{x}}$ are the electron and exciton in-plane effective masses, respectively) we can decompose the exciton wave function into an envelope function, $\phi\left(r_{\|}, z_{e}, z_{h}\right)$, and a free motion part related to the in-plane CM coordinate. Denoting $\mathbf{k}_{\mathrm{x}}$ as the inplane CM momentum we find

$$
\begin{aligned}
\Phi_{\mathbf{k}_{1}, \mathbf{k}_{2}}\left(z_{e}, z_{h}\right)= & \frac{1}{A} \int d^{2} R_{\|} d^{2} r_{\|} \phi\left(r_{\|}, z_{e}, z_{h}\right) \mathrm{e}^{\mathrm{i} \mathbf{k}_{\mathrm{x}} \cdot \mathbf{R}_{\|}} \times \\
& \mathrm{e}^{-\mathrm{i}\left[\mathbf{R}_{\|} \cdot\left(\mathbf{k}_{1}+\mathbf{k}_{2}\right)+\mathbf{r}_{\|} \cdot\left(\beta \mathbf{k}_{1}-\alpha \mathbf{k}_{2}\right)\right]} \\
= & \delta\left(\mathbf{k}_{\mathrm{x}}-\mathbf{k}_{1}-\mathbf{k}_{2}\right) \phi_{\mathbf{k}_{1}-\alpha \mathbf{k}_{\mathrm{x}}}\left(z_{e}, z_{h}\right)(\mathrm{A}-2)
\end{aligned}
$$

We use the simplest exciton wave function for the exciton ground state: $\phi\left(r_{\|}, z_{e}, z_{h}\right)=\mathcal{N} \chi_{e}\left(z_{e}\right) \chi_{h}\left(z_{h}\right) \mathrm{e}^{-r_{\|} / \lambda}$, where $\mathcal{N}$ is a normalization factor and $\lambda$ is a variational parameter associated with the exciton Bohr radius in the QW, whichis fixed by maximizing the binding energy of the exciton 24. We note that the use of a wave function separable in $z$ and $r_{\|}$, facilitates considerably the calculation of the scattering matrix elements, although it is strictly justifiable only for narrow well structures. Assuming perfect confinement of electrons and holes in the QW, and taking the $z$ axis origin in the center of the QW, the confinement functions are:

$$
\chi_{e}\left(z_{e}\right)=\chi_{h}\left(z_{h}\right)= \begin{cases}\cos (\pi z / L) & |z| \leq L / 2 \\ 0 & |z|>L / 2\end{cases}
$$

This approximation is quite accurate for almost all practical thicknesses $L$. Calculating the binding energy of the $1 \mathrm{~S}$ exciton in a GaAs QW of width $L=200 \AA$, we find $E_{b}=6.73 \mathrm{meV}, \lambda=150 \AA\left(E_{b}=7.49 \mathrm{meV}, \lambda=133 \AA\right)$ for the heavy-hole (light-hole) exciton. These results, obtained with our single parameter trial function, are quite close to those obtained in 28 , which took into account finite QW barriers, and anisotropic masses.

Combining the first terms from Eqs. (II.4), the direct term reads

$$
\begin{aligned}
V_{\text {dir }}(q)= & \frac{32 \lambda^{2} e^{2}}{\epsilon_{0} A L^{3} q} \int_{-L / 2}^{L / 2} d z_{e} d z_{h} d z_{c} \int d^{2} k_{1} \mathrm{e}^{-q\left|z_{e}-z_{c}\right|} \times \\
& {\left[g\left(\lambda\left|\beta \mathbf{q}-\mathbf{k}_{1}\right|\right)-g\left(\lambda\left|\alpha \mathbf{q}+\mathbf{k}_{1}\right|\right)\right] g\left(\lambda k_{1}\right) \times } \\
& \cos ^{2}\left(\pi z_{e} / L\right) \cos ^{2}\left(\pi z_{h} / L\right) \cos ^{2}\left(\pi z_{c} / L\right), \quad(\mathrm{A}-4
\end{aligned}
$$

where $g(\lambda q)$ was defined in Eq. (II.6). We note that the electron wave functions in the QW plane are absent, as they contribute, together with the in-plane center of mass part of the exciton wave function, a fixed phase factor which is unimportant for our purposes. The momentum integral is a simple convolution and can be readily evaluated

$$
\begin{aligned}
& \int d^{2} k_{1}\left[g\left(\lambda\left|\beta \mathbf{q}-\mathbf{k}_{1}\right|\right)-g\left(\lambda\left|\alpha \mathbf{q}+\mathbf{k}_{1}\right|\right)\right] g\left(\lambda k_{1}\right) \\
= & \frac{1}{4 \pi^{2}} \int d^{2} \rho \tilde{g}^{2}(\rho / \lambda)[\exp (\mathrm{i} \boldsymbol{\rho} \cdot \beta \mathbf{q})-\exp (\mathrm{i} \boldsymbol{\rho} \cdot \alpha \mathbf{q})] \\
= & \frac{\pi}{2 \lambda^{2}}[g(\lambda \beta q / 2)-g(\lambda \alpha q / 2)]
\end{aligned}
$$

where

$$
\tilde{g}(\rho / \lambda)=\frac{2 \pi}{\lambda^{2}} \mathrm{e}^{-\rho / \lambda}
$$

is the exciton spatial wave function in the QW plane. In order to evaluate the $z$ integrals, we change to the coordinates

$$
z=z_{e}-z_{c} \quad ; \quad z^{\prime}=\frac{1}{2}\left(z_{e}+z_{c}\right)
$$

which implies the following change in the integration limits:

$$
\begin{aligned}
\int_{-L / 2}^{L / 2} d z_{e} \int_{-L / 2}^{L / 2} d z_{c} & =\int_{-L / 2}^{0} d z^{\prime} \int_{-\left(L+2 z^{\prime}\right)}^{L+2 z^{\prime}} d z \\
& +\int_{0}^{L / 2} d z^{\prime} \int_{-\left(L-2 z^{\prime}\right)}^{L-2 z^{\prime}} d z
\end{aligned}
$$

and performing the integrations results in the direct term, given by Eq. (II.5)

\section{Appendix B}

In this Appendix we present an analytical calculation of the angular part of the exchange integral. This calculation, while quite complicated, is worth the trouble for two reasons. The first is, obviously, a major reduction in the time consumed by the numerical computation, making it feasible. As a subsidiary benefit, the partially analytical calculation removes some convergence problems encountered in the previously used numerical algorithm.

Using dimensionless momenta (e.g., by writing $q$ we mean $\lambda q)$ the exchange integral reads 


$$
\begin{aligned}
I_{\mathrm{exc}}(\Delta k, q, \theta)= & -4 \pi \int d k_{1} k_{1} h\left(k_{1}\right) d \phi\left\{\left[1+\left(\mathbf{k}_{1}-\Delta \mathbf{k}+\mathbf{q}\right)^{2}\right]^{-3 / 2}-\left[1+(\mathbf{q}-\Delta \mathbf{k})^{2}\right]^{-3 / 2}\right\} \times \\
& {\left[1+\left(\mathbf{k}_{1}-\Delta \mathbf{k}+\alpha \mathbf{q}\right)^{2}\right]^{-3 / 2} \equiv-4 \pi \int d k_{1} k_{1} h\left(k_{1}\right)\{(I)+(I I)\} }
\end{aligned}
$$

where $\theta$ is the angle between $\mathbf{q}$ and $\Delta \mathbf{k}$ and $\phi$ is the angle between $\mathbf{k}_{1}$ and $\Delta \mathbf{k}$. We shall work out separately the two parts of Eq. (B-1).

The first part is given by

$$
\begin{aligned}
(I)= & \int_{0}^{2 \pi} d \phi[a d+(b d+a e) \cos \phi- \\
& c(d+a / \alpha) \sin \phi-c(e+b / \alpha) \sin \phi \cos \phi+ \\
& \left.b e \cos ^{2} \phi+c^{2} / \alpha \sin ^{2} \phi\right]^{-3 / 2}
\end{aligned}
$$

where we have defined

$$
\begin{aligned}
a & =1+k_{1}^{2}+\Delta k^{2}+\alpha^{2} q^{2}-2 \alpha q \Delta k \cos \theta \\
b & =2 k_{1}(\alpha q \cos \theta-\Delta k) \\
c & =2 \alpha q k_{1} \sin \theta \\
d & =1+k_{1}^{2}+\Delta k^{2}+q^{2}-2 q \Delta k \cos \theta \\
e & =2 k_{1}(q \cos \theta-\Delta k) .
\end{aligned}
$$

Transforming $\phi \rightarrow \phi^{\prime}=\phi-\pi$ and then to $z=\tan (\phi / 2)$ we have

$$
\begin{aligned}
(I)= & 2 \int_{-\infty}^{\infty} d z\left(1+z^{2}\right)^{2}[(a-b)(d-e)+ \\
& 2 c(d-e+a / \alpha-b / \alpha) z+2\left(a d-b e+2 c^{2} / \alpha\right) z^{2}+ \\
& \left.2 c(d+e+a / \alpha+b / \alpha) z^{3}+(a+b)(d+e) z^{4}\right]^{-3 / 2} .
\end{aligned}
$$

Finding the roots of this 4 th order polynomial we have

$$
\begin{aligned}
(I)= & 2 \int_{-\infty}^{\infty} d z\left(1+z^{2}\right)^{2}\left[\left(z^{2}+2 A z+B\right) \times\right. \\
& \left.\left(z^{2}+2 C z+D\right)(a+b)(d+e)\right]^{-3 / 2}
\end{aligned}
$$

where

$$
A=\frac{c}{a+b} \quad ; \quad B=\frac{a-b}{a+b} \quad ; \quad C=\frac{c}{\alpha(d+e)} \quad ; \quad D=\frac{d-e}{d+e} .
$$

We note for later use that $A, B, C, D$ obey the inequalities

$$
B>0 ; D>0 ; B-A^{2}>0 ; D-C^{2}>0 .
$$

One can reduce the polynomial in Eq. (B-4) to a product of two quadratic binomial forms, by making use of the following transformation

with $p, q$ given by

$$
z=\frac{p+q y}{1+y}
$$

$$
p, q=\frac{1}{2} \frac{D-B}{A-C} \pm \sqrt{\frac{1}{4}\left(\frac{D-B}{A-C}\right)^{2}+\frac{A D-B C}{A-C}}
$$

(note that we assume $A \neq C$, deferring for later the treatment in this special case). Noticing that $p$ and $q$ always have opposite signs, we choose $q<0$ and divide the integral into

$$
\int_{-\infty}^{\infty} d z=\int_{-\infty}^{0} d z+\int_{0}^{\infty} d z
$$

where the limit $z=\infty$ corresponds to $y=-1$ and the limit $z=-\infty$ corresponds to $y=-1$ with $q$ and $p$ interchanged. The integral now reads

$$
(I)=\mathcal{N}\left\{\int_{-p / q}^{-1} d y \frac{\left[y^{2}\left(1+q^{2}\right)+2 y(1+p q)+1+p^{2}\right]^{2}}{\left[\left(y^{2}+s^{2}\right)\left(y^{2}+t^{2}\right)\right]^{3 / 2}}-\frac{1}{(s t)^{3}} \int_{-1}^{-q / p} d y \frac{\left[y^{2}\left(1+p^{2}\right)+2 y(1+p q)+1+q^{2}\right]^{2}}{\left[\left(y^{2}+1 / s^{2}\right)\left(y^{2}+1 / t^{2}\right)\right]^{3 / 2}}\right\}
$$

where

$$
s^{2}=\frac{p^{2}+2 A p+B}{q^{2}+2 A q+B} \quad ; \quad t^{2}=\frac{p^{2}+2 C p+D}{q^{2}+2 C q+D}
$$

and

$$
\mathcal{N}=\frac{2(q-p)}{\left[\left(q^{2}+2 A q+B\right)\left(q^{2}+2 C q+D\right)(a+b)(d+e)\right]^{3 / 2}}
$$


Making the transformation $y=s \tan \psi$ in the first integral and $y=\frac{1}{s} \tan \psi$ in the second, we find

$$
\begin{aligned}
(I)= & \frac{\mathcal{N}}{s^{2} t^{3}}\left\{\int_{\tan ^{-1}(-p / s q)}^{\tan ^{-1}(-1 / s)} d \psi \frac{\left[(q s \sin \psi+p \cos \psi)^{2}+(s \sin \psi+\cos \psi)^{2}\right]^{2}}{\left(1-k^{2} \sin ^{2} \psi\right)^{3 / 2}}-\right. \\
& \left.s t^{3} \int_{\tan ^{-1}(-s)}^{\tan ^{-1}(-q s / p)} d \psi \frac{\left[(p / s \sin \psi+q \cos \psi)^{2}+(1 / s \sin \psi+\cos \psi)^{2}\right]^{2}}{\left(1-\tilde{k}^{2} \sin ^{2} \psi\right)^{3 / 2}}\right\},
\end{aligned}
$$

where we have defined

$$
k^{2}=1-s^{2} / t^{2} \quad ; \quad \tilde{k}^{2}=1-t^{2} / s^{2} .
$$

Changing $\psi \longrightarrow \pi / 2-\psi$ in the second integral, and using $\tan ^{-1} s+\cot ^{-1} s=\pi / 2$ we find that the second integral exactly matches the first except that its upper limit is larger by $\pi$; thus we are left with

$$
(I)=-\frac{\mathcal{N}}{s^{5}} \int_{-\tan ^{-1} s}^{\pi-\tan ^{-1} s} d \psi \frac{\left[\left(1+p^{2}\right) \sin ^{2} \psi+s^{2}\left(1+q^{2}\right) \cos ^{2} \psi+s(1+p q) \sin 2 \psi\right]^{2}}{\left(1-\tilde{k}^{2} \sin ^{2} \psi\right)^{3 / 2}} .
$$

This integral can be evaluatedusing incomplete elliptic integrals (see p. 201 in 29). Furthermore, one can exploit the functional relations (p. 911 in 29 )

$$
\begin{array}{ll}
F(-\phi, k)=-F(\phi) \quad ; \quad F(n \pi-\phi, k)=2 \mathbf{K}(k)-F(\phi, k) \\
E(-\phi, k)=-E(\phi) \quad ; \quad E(n \pi-\phi, k)=2 \mathbf{E}(k)-E(\phi, k)
\end{array}
$$

where $F(\phi, k), E(\phi, k)$ denote incomplete elliptic integrals of the first and second kind, respectively, and $\mathbf{K}(k), \mathbf{E}(k)$ denote complete elliptic integrals of the first and second kind, respectively. The final result is

$$
\begin{aligned}
(I)= & -\frac{2 \mathcal{N}}{s\left(s^{2}-t^{2}\right)^{2}}\left\{\left[\left(s^{2}+t^{2}\right) \mathbf{E}(\tilde{k})-2 t^{2} \mathbf{K}(\tilde{k})\right]\left[\frac{1}{t^{2}}\left(1+p^{2}\right)^{2}+\left(1+q^{2}\right)^{2} s^{2}\right]-\right. \\
& \left.2\left(3+3 p^{2} q^{2}+4 p q+p^{2}+q^{2}\right)\left[2 s^{2} \mathbf{E}(\tilde{k})-\left(s^{2}+t^{2}\right) \mathbf{K}(\tilde{k})\right]\right\} .
\end{aligned}
$$

For imaginary values of $\tilde{k}$, the result is the same as Eq. (B-7) with the interchanges $s \longleftrightarrow t$ and $\tilde{k} \longrightarrow k, k$ being defined in (B-6).

We now return to examine two special cases which cannot be treated using (B-7). The first case corresponds to $A=C, B \neq D$. Here we have

$$
p= \pm \infty \quad ; \quad q=-A .
$$

Although $s, t=\infty, \tilde{k}$ is still finite, taking the value

$$
\tilde{k}^{2}=\frac{D-B}{D-A^{2}},
$$

thus, enabling the use of (B-7) with some modifications. Remembering the inequalities $(\mathrm{B}-5)$ we note that the sign of $\tilde{k}^{2}$ is determined by the sign of $D-B$. We obtain, for $D>B$

$$
\begin{aligned}
& (I)=\frac{4 \sqrt{D-A^{2}}}{[(a+b)(d+e)]^{3 / 2}(D-B)^{2}} \times \\
& \left\{[ 1 + \frac { ( 1 + A ^ { 2 } ) ^ { 2 } } { ( B - A ^ { 2 } ) ( D - A ^ { 2 } ) } ] \left[\left(1+D-A^{2}\right) \mathbf{E}(\tilde{k})-\right.\right. \\
& \left.2 \mathbf{K}(\tilde{k})]-2\left(3 A^{2}+1\right)\left[2 \mathbf{E}(\tilde{k})-\left(1+\frac{B-A^{2}}{D-A^{2}}\right) \mathbf{K}(\tilde{k})\right]\right\} .
\end{aligned}
$$

For $D<B$ the result is the same as Eq. ( $\mathrm{B}-8)$ with the interchanges $B \longleftrightarrow D$ and $\tilde{k} \longrightarrow k$.

The second special case corresponds to $A=C, B=D$. Here the integral $(\mathrm{B}-4)$ reduces to

$$
(I)=\frac{2}{[(a+b)(d+e)]^{3 / 2}} \int_{-\infty}^{\infty} d z \frac{\left(1+z^{2}\right)^{2}}{\left(z^{2}+2 A z+B\right)^{3}} .
$$

This integral can be easily evaluated (see p. 81 in 29 ) and the result is

$$
(I)=\frac{\pi}{4} \frac{3+4 A^{2}+2 B+3 B^{2}}{[(a+b)(d+e)]^{3 / 2}\left(B-A^{2}\right)^{5 / 2}} .
$$

Finally, we evaluate the second part of the exchange integral, appearing in Eq. (B-1). We have

$$
(I I)=\frac{1}{\left(d-k_{1}^{2}\right)^{3 / 2}} \int_{0}^{2 \pi} \frac{d \phi}{(a+b \cos \phi-c \sin \phi)^{3 / 2}},
$$

where $a, b, c, d$ where defined in (B-4). Using the transformation $\phi=2 \psi+\beta$, where $\tan \beta=-c / b$, we have

$$
(I I)=\frac{2}{\left(d-k_{1}^{2}\right)^{3 / 2}} \int_{-\beta / 2}^{\pi-\beta / 2} \frac{d \psi}{\left(a+p-2 p \sin ^{2} \psi\right)^{3 / 2}},
$$


where $p=\sqrt{b^{2}+c^{2}}$. This integral can be evaluated using the elliptic integral of second kind, resulting in

$$
(I I)=\frac{4 \sqrt{a+p}}{\left(d-k_{1}^{2}\right)^{3 / 2}\left(a^{2}-p^{2}\right)} \mathbf{E}\left(\frac{2 p}{a+p}\right) .
$$

\section{Appendix C}

In this Appendix we present the calculation of the trion binding energy, based on the trial wave function given in
Eq. (III.1). It is useful to work with the Fourier transformed trion wave function (III.6), where we have for the two spin configurations, explicitly

$$
\begin{aligned}
\phi_{\mathbf{k}_{1}, \mathbf{k}_{2}}^{\mathrm{tr}}\left(z_{1}, z_{2}, z_{h}\right)= & \mathcal{N}_{\mathrm{tr}} \chi\left(z_{1}\right) \chi\left(z_{2}\right) \chi\left(z_{h}\right) \times \\
& {\left[\phi_{\mathbf{k}_{1}} \phi_{\mathbf{k}_{2}}^{\prime} \pm \phi_{\mathbf{k}_{1}}^{\prime} \phi_{\mathbf{k}_{2}}\right] . }
\end{aligned}
$$

The effective mass Hamiltonian is given by

$$
H=\sum_{i=1}^{2}\left[-\frac{\hbar^{2}}{2 m_{e}}\left(\nabla_{\mathbf{r}_{i}}^{2}+\partial_{z_{i}}^{2}\right)-\frac{e^{2}}{\epsilon_{0} \sqrt{\left(\mathbf{r}_{i}-\mathbf{r}_{h}\right)^{2}+\left(z_{i}-z_{h}\right)^{2}}}\right]+-\frac{\hbar^{2}}{2 m_{e}}\left(\sigma \nabla_{\mathbf{r}_{h}}^{2}+\sigma_{z} \partial_{z_{h}}^{2}\right)+\frac{e^{2}}{\epsilon_{0} \sqrt{\left(\mathbf{r}_{1}-\mathbf{r}_{2}\right)^{2}+\left(z_{1}-z_{2}\right)^{2}}}
$$

where $\sigma=m_{e} / m_{h}^{\|}, \sigma_{z}=m_{e} / m_{h}^{z}$ are the electron-hole effective mass ratios in the plane and $z$ directions, respectively. Transforming to CM and relative coordinates in the QW plane:

$$
\begin{aligned}
\mathbf{R}_{\mathrm{cm}} & =\frac{\sigma\left(\mathbf{r}_{1}+\mathbf{r}_{2}\right)+\mathbf{r}_{h}}{1+2 \sigma} \\
\mathbf{r}_{\mathrm{ih}} & =\mathbf{r}_{i}-\mathbf{r}_{h}, \quad i=1,2
\end{aligned}
$$

and neglecting CM motion which does not contribute to the binding energy, we have

$$
H=\sum_{i=1}^{2} H_{0}\left(\mathbf{r}_{\mathrm{ih}}, z_{i}, z_{h}\right)+H_{12}\left(\mathbf{r}_{1 \mathrm{~h}}, z_{1}, \mathbf{r}_{2 \mathrm{~h}}, z_{2}\right)
$$

where

$$
\begin{aligned}
H_{0}\left(\mathbf{r}_{\mathrm{ih}}, z_{i}, z_{h}\right) & =-(1+\sigma) \nabla_{\mathbf{r}_{\mathrm{ih}}}^{2}-\frac{\sigma_{z}}{2} \partial_{z_{i}}^{2}-\frac{2}{\sqrt{r_{\mathrm{ih}}^{2}+\left(z_{i}-z_{h}\right)^{2}}} \\
H_{12}\left(\mathbf{r}_{1 \mathrm{~h}}, z_{1}, \mathbf{r}_{2 \mathrm{~h}}, z_{2}\right) & =-2 \sigma \nabla_{r_{1 \mathrm{~h}}} \cdot \nabla_{r_{2 \mathrm{~h}}}+\frac{2}{\sqrt{\left(\mathbf{r}_{1 \mathrm{~h}}-\mathbf{r}_{2 \mathrm{~h}}\right)^{2}+\left(z_{1}-z_{2}\right)^{2}}}
\end{aligned}
$$

and we employed natural units of length and energy, namely, bulk effective Bohr radius and Rydberg. The expectation value of the trion energy is given by

$$
E=\left\langle\phi^{\mathrm{tr}}|H| \phi^{\mathrm{tr}}\right\rangle=\frac{\mathcal{I}_{1} \pm 2 \kappa \mathcal{I}_{2}+\mathcal{J}_{1} \pm \mathcal{J}_{2}}{1 \pm \kappa^{2}}
$$

where $\kappa$ was defined in Eq. (III.4), and the upper (lower) signs apply to the singlet (triplet) spin configuration. The various terms appearing in Eq. (C-5) are calculated in the in-plane Fourier space, resulting in simple 2D convolutions, similar to those in appendix A. The results are:

$$
\begin{aligned}
\mathcal{I}_{1}= & \left(\frac{2}{L}\right)^{2}\left[\left\langle\phi \chi_{1} \chi_{h}\left|H_{0}\right| \phi \chi_{1} \chi_{h}\right\rangle+\left\langle\phi^{\prime} \chi_{1} \chi_{h}\left|H_{0}\right| \phi^{\prime} \chi_{1} \chi_{h}\right\rangle\right]=\left(\frac{\pi}{L}\right)^{2}\left(2+\sigma_{z}\right)+ \\
& {\left[\frac{1}{\lambda^{2}}(1+\sigma)-\lambda^{3}\left(\frac{2}{L}\right)^{2} \int_{0}^{\infty} d q q h(\lambda q) g(\lambda q / 2)\right]+\left[\lambda \rightarrow \lambda^{\prime}\right] } \\
\mathcal{I}_{2}= & \left(\frac{2}{L}\right)^{2}\left\langle\phi \chi_{1} \chi_{h}\left|H_{0}\right| \phi^{\prime} \chi_{1} \chi_{h}\right\rangle=\frac{\kappa}{2}\left(\frac{\pi}{L}\right)^{2}\left(2+\sigma_{z}\right)+\frac{4(1+\sigma)}{\left(\lambda+\lambda^{\prime}\right)^{2}}-\left(\lambda+\lambda^{\prime}\right)\left(\frac{\tilde{\lambda} \kappa}{L}\right)^{2} \int_{0}^{\infty} d q q h(\tilde{\lambda} q) g(\tilde{\lambda} q)\left(\mathrm{C}-\left(\mathrm{C}^{2}-{ }^{2}\right.\right. \\
\mathcal{J}_{1}= & \left(\frac{2}{L}\right)^{2}\left\langle\phi_{1} \chi_{1} \phi_{2}^{\prime} \chi_{2}\left|H_{12}\right| \phi_{1} \chi_{1} \phi_{2}^{\prime} \chi_{2}\right\rangle=2 \lambda^{3}\left(\frac{2}{L}\right)^{2} \int_{0}^{\infty} d q q h(2 \lambda q) g(\lambda q / 2) g\left(\lambda^{\prime} q / 2\right) \\
\mathcal{J}_{2}= & \left(\frac{2}{L}\right)^{2}\left\langle\phi_{1} \chi_{1} \phi_{2}^{\prime} \chi_{2}\left|H_{12}\right| \phi_{1}^{\prime} \chi_{1} \phi_{2} \chi_{2}\right\rangle=2 \kappa^{2} \tilde{\lambda}^{3}\left(\frac{2}{L}\right)^{2} \int_{0}^{\infty} d q q h(2 \tilde{\lambda} q) g^{2}(\tilde{\lambda} q)
\end{aligned}
$$


In Eqs. (C-6), $h(\lambda q), g(\lambda q)$ are the functions defined in Eq. (II.6), and we have denoted $\tilde{\lambda}=\lambda \lambda^{\prime} /\left(\lambda+\lambda^{\prime}\right)$. We note that in the $2 \mathrm{D}$ limit $(L \rightarrow 0)$, eliminating the kinetic energy term in the $z$ direction, Eqs. (C-6) reduce to

$$
\begin{aligned}
& \mathcal{I}_{1}=\left(\frac{1}{\lambda^{2}}+\frac{1}{\lambda^{\prime 2}}\right)(1+\sigma)-4\left(\frac{1}{\lambda}+\frac{1}{\lambda^{\prime}}\right) \\
& \mathcal{I}_{2}=\frac{4(1+\sigma)}{\left(\lambda+\lambda^{\prime}\right)^{2}}-\frac{8}{\lambda+\lambda^{\prime}} \\
& \mathcal{J}_{1}=\left\{\begin{array}{ll}
\frac{\lambda \kappa}{\left(\lambda^{\prime}-\lambda^{2}\right.} \\
\frac{\lambda^{\prime} \kappa}{\left(\lambda^{\prime}-\lambda\right)^{2}} \\
\left.\frac{3 \pi}{4 \lambda},\left(1+\frac{\lambda^{\prime 2}}{\lambda^{2}}\right) \mathbf{E}\left(1-\lambda^{2} / \lambda^{\prime 2}\right)-2 \mathbf{K}\left(1-\lambda^{2} / \lambda^{\prime 2}\right)\right], & \lambda<\lambda^{\prime} \\
\lambda^{\prime 2}
\end{array}\right) \begin{array}{ll}
\left.\mathbf{E}\left(1-\lambda^{\prime 2} / \lambda^{2}\right)-2 \mathbf{K}\left(1-\lambda^{\prime 2} / \lambda^{2}\right)\right], & \lambda>\lambda^{\prime} \\
\mathcal{J}_{2} & =\frac{3 \pi \kappa}{2\left(\lambda+\lambda^{\prime}\right)}
\end{array}
\end{aligned}
$$

where $\mathbf{K}, \mathbf{E}$ are the complete elliptic integrals of the first and second kind, respectively. These closed form expressions coincide with the integral expressions of Sandler and Proettd23, for $\sigma \rightarrow 0$ and vanishing magnetic field (note a misprint in Eq. A6 of this paper).

In order to maximize the trion binding energy, we must first write the expectation value of the exciton energy:

$$
\begin{aligned}
E_{0}= & \frac{1}{\lambda_{0}}(1+\sigma)+\left(\frac{\pi}{L}\right)^{2}\left(1+\sigma_{z}\right)- \\
& \frac{4 \lambda_{0}^{3}}{L^{2}} \int_{0}^{\infty} d q q h\left(\lambda_{0} q\right) g\left(\lambda_{0} q / 2\right),
\end{aligned}
$$

resulting in the $1 \mathrm{~S}$ exciton Bohr radius and binding energy values stated in Appendix A. Note that in the 2D limit we have

$$
E_{0}(L \rightarrow 0)=\frac{1+\sigma}{\lambda_{0}^{2}}-\frac{4}{\lambda_{0}} .
$$

Minimizing Eq. (C-9) in the $\sigma \rightarrow 0$ limit, results in $\lambda_{0}=$ $a_{\mathrm{B}} / 2, E_{0}=-4 \mathrm{Ry}$, as expected. The trion binding energy with respect to the exciton is related to the trion and exciton energies through

$$
E_{b}^{\operatorname{tr}}=E-E_{0}-E_{\mathrm{e}}
$$

where $E_{\mathrm{e}}=(\pi / L)^{2}$ is the confined electron energy, in our infinite barrier model. We note that the kinetic energy terms in the $z$ direction cancel out in the expression for the trion binding energy, so they can be removed from the calculation. Maximizing Eq. (C-10) with respect to the two variational parameters $\lambda, \bar{\lambda}^{\prime}$, we finally find the results given in section III, where we used the GaAs bulk values of the effective Bohr radius $\left(a_{B}=100 \AA\right)$, and Rydberg (5.7meV).
* Electronic address: ramon@physics.technion.ac.il.

1 R. C. C. Leite, J. Shah, and J. P. Gordon, Phys. Rev. Lett. 23, 1332 (1969).

2 L. Schultheis, J. Kuhl, A. Honold and C. W. Tu, Phys. Rev. Lett. 57, 1635 (1986).

3 A. Honold, L. Schultheis, J. Kuhl and C. W. Tu, Phys. Rev. B 40, 6442 (1989).

4 V. Capozi, L. Pavesi, and J. L. Staehli, Phys. Rev. B 47, 6340 (1993).

${ }^{5}$ K. Matsuda, M. Hirooka, and S. Sunakawa, Prog. Theor. Phys. 54, 79 (1975); S. G. Elkomoss and G. Munschy, J. Phys. Chem. Solids 38, 557 (1977); 40, 431 (1979); 42, 1 (1981).

6 Y.-P. Feng and H. N. Spector, J. Phys. Chem. Solids 48, 593 (1987); Superlattices and Microstructures 3, 459 (1987) ; Y.-P. Feng Z. Huang, and H. N. Spector, J. Phys. Chem. Solids 50, 117 (1989); T. S. Koh, Y.-P. Feng and H. N. Spector, J. Appl. Phys. 81, 2236 (1997).

7 G. Ramon, R. Rapaport, A. Qarry, E. Cohen, A. Mann, Arza Ron, and L. N. Pfeiffer, Phys. Rev. B 65, 085323
(2002)

8 M. A. Lampert, Phys. Rev. Lett. 1, 450 (1958).

9 B. Stèbè and A. Ainane, Superlatt. Microstruct. 5, 545 (1989).

${ }^{10}$ K. Kheng, R. T. Cox, Y. Merle d'Aubigné, F. Bassani, K. Saminadayar, and S. Tatarenko, Phys. Rev. Lett. 71, 1752 (1993).

11 G. Finkelstein, H. Shtrikman, and I. Bar-Joseph, Phys. Rev. Lett. 74, 976 (1995); A. J. Shields, M. Pepper, D. A. Ritchie, M. Y. Simmons, and G. A. C. Jones, Phys. Rev. B 51, 18049 (1995); Arza Ron, H. W. Yoon, M. D. Sturge, A. Manassen, E. Cohen, and L. N. Pfeiffer, Sol. State Comm. 97, 741 (1996).

12 A. I. Bobrysheva, M. V. Grodetskii, and V. T. Z. Zyukov, J. Phys. C 16, 5723 (1983); A. Thilagam, Phys. Rev. B 55, 7804 (1997).

13 B. Stèbè, G. Munschy, L. Stauffer, F. Dujardin, and J. Murat, Phys. Rev. B 56, 12454 (1997).

14 J. R. Chapman, N. F. Johnson, and V. N. Nicopoulos, Phys. Rev. B 55, R10221 (1997); D. M. Whittaker and 
A. J. Shields, ibid 56, 15185 (1997); A. Wójs, J. J. Quinn, and P. Hawrylak, ibid 62, 4630 (2000).

15 S. Glasberg, G. Finkelstein, H. Shtrikman, and I. BarJoseph, Phys. Rev. B 59, R10425 (1999).

16 Y. Yayon, M. Rappaport, V. Umansky, and I. Bar-Joseph, Phys. Rev. B 64, 081308(R) (2001).

17 A. Manassen, E. Cohen, Arza Ron, E. Linder, and L. N. Pfeiffer, Phys. Rev. B 54, 10609 (1996).

18 F. Tassone and Y. Yamamoto Phys. Rev. B 59, 10830 (1999).

19 C. Ciuti, V. Savona, C. Piermarocchi, A. Quattropani and P. Schwendimann, Phys. Rev. B 58, 7926 (1998).

20 The angle between $\mathbf{k}_{\mathrm{x}}$ and $\mathbf{k}_{\mathrm{e}}$ is thus given by $\gamma \pm \delta$, where it is noted that since one can make the transformation $\delta \rightarrow 2 \pi-\delta$, the result of the integration over $\delta$ is indifferent to the sign choice in $\cos (\gamma \pm \delta)$.

21 D. Huang, J.-I. Chyi, and H. Morkoç, Phys. Rev. B 42, 5147 (1990).

22 S. Chandrasekhar, Astrophys. J. 100, 176 (1944); H. A. Bethe and E. E. Salpeter, Quantum Mechanics of One- and Two-Electron Atoms (Plenum, New York, 1957).

23 N. P. Sandler and C. R. Proetto, Phys. Rev. B 46, 7707 (1992).

24 G. Bastard, Wave Mechanics Applied to Semiconductor Heterostructures (Les Editions de Physique CNRS, Paris, 1988).

${ }^{25}$ R. Rapaport, A. Qarry E. Cohen, Arza Ron, E. Linder, and L. N. Pfeiffer, in Proceedings of the 25th International
Conference on the Physics of Semiconductors, edited by N. Miura and T. Ando (Springer-Verlag, 2001), p. 679.

26 J. Christen, and D. Bimberg, Phys. Rev. B 42, 7213 (1990).

27 V. Savona, L. C. Andreani, P. Schwendimann and A. Quattropani, Solid State Comm. 93, 733 (1995).

28 M. Grundmann and D. Bimberg, Phys. Rev. B 38, 13486 (1988).

29 I. S. Gradshteyn and I. M. Ryzhik, Tables of Integrals, Series, and Products (Academic press, San Diego, 1994).

30 R. Rapaport, R. Harel, E. Cohen, Arza Ron, E. Linder and L. N. Pfeiffer, Phys. Rev. Lett. 84, 1607 (2000); Phys. Stat. Sol. a 178, 481 (2000).

31 P. G. Savvidis, J. J. Baumberg, R. M. Stevenson, M. S. Skolnick, D. M. Whittaker, and J. S. Roberts, Phys. Rev. Lett. 84, 1547 (2000); J. J. Baumberg, P. G. Savvidis, R. M. Stevenson, A. I. Tartakovskii, M. S. Skolnick, D. M. Whittaker, and J. S. Roberts, Phys. Rev. B 62 R16247 (2000); P. G. Savvidis, C. Ciuti, J. J. Baumberg, D. M. Whittaker, M. S. Skolnick, and J. S. Roberts, ibid. 64075311 (2001).

32 Le Si Dang, D. Heger, R. André, F. Bœuf, and R. Romestain, Phys. Rev. Lett. 81, 3920 (1998); P. Senellart, J. Bloch, B. Sermage, and J. Y. Marzin, ibid. 62, R16263 (2000); R. Huang, F. Tassone, and Y. Yamamoto, ibid. 61, R7854 (2000). 